Pacific Northwest

National Laboratory

Operated by Battelle for the

U.S. Department of Energy

\section{Description of the RDCDS \\ Meteorological Component}

MS Pekour

LK Berg

October 2007

Prepared for the U.S. Department of Energy under Contract DE-AC05-76RL01830 


\title{
DISCLAIMER
}

This report was prepared as an account of work sponsored by an agency of the United States Government. Neither the United States Government nor any agency thereof, nor Battelle Memorial Institute, nor any of their employees, makes any warranty, express or implied, or assumes any legal liability or responsibility for the accuracy, completeness, or usefulness of any information, apparatus, product, or process disclosed, or represents that its use would not infringe privately owned rights. Reference herein to any specific commercial product, process, or service by trade name, trademark, manufacturer, or otherwise does not necessarily constitute or imply its endorsement, recommendation, or favoring by the United States Government or any agency thereof, or Battelle Memorial Institute. The views and opinions of authors expressed herein do not necessarily state or reflect those of the United States Government or any agency thereof.

\author{
PACIFIC NORTHWEST NATIONAL LABORATORY \\ operated by \\ BATTELLE \\ for the \\ UNITED STATES DEPARTMENT OF ENERGY \\ under Contract DE-AC05-76RL01830
}

Printed in the United States of America
Available to DOE and DOE contractors from the Office of Scientific and Technical Information,
P.O. Box 62, Oak Ridge, TN 37831-0062;
ph: (865) 576-8401
fax: $(865)$ 576-5728
email: reports@adonis.osti.gov

\begin{abstract}
Available to the public from the National Technical Information Service, U.S. Department of Commerce, 5285 Port Royal Rd., Springfield, VA 22161 ph: (800) 553-6847 fax: $(703) 605-6900$ email: orders@ntis.fedworld.gov online ordering: http://www.ntis.gov/ordering.htm
\end{abstract}

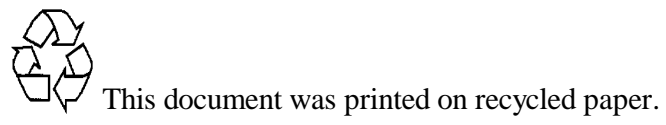


PNNL-17106

\title{
Description of the RDCDS Meteorological Component
}

\author{
M. S. Pekour \\ L. K. Berg
}

October 2007

Prepared for the U.S. Department of Homeland Security under a Related Services Agreement with the U.S. Department of Energy under Contract DE-AC05-76RL01830

Pacific Northwest National Laboratory Richland, Washington 99352 



\section{Summary}

This report provides a detailed description of the Rapidly Deployable Chemical Defense System (RDCDS) Meteorological Component. The Meteorological Component includes four surface meteorological stations, miniSODAR, laptop computers, and communications equipment. This report describes the equipment that is used, explains the operation of the network, and gives instructions for setting up the Component and replacing defective parts. A detailed description of operation and use of the individual sensors, including the data loggers is not covered in the current document, and the interested reader should refer to the manufacturer's documentation.

This manual was written for scientists or technicians who are tasked with the deployment and operation of the RDCDS network. The reader should have 1) experience working with laptop computers operating with the Windows XP operating system and 2) the technical background necessary to ensure that proper locations are selected for the individual meteorological instruments. 



\section{Contents}

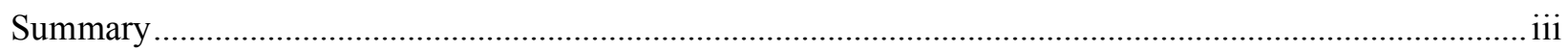

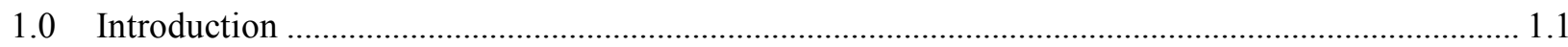

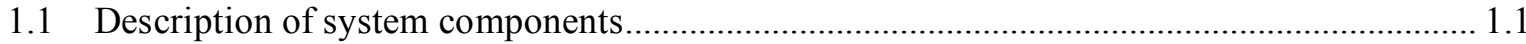

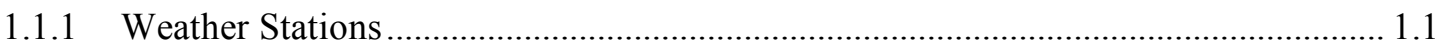

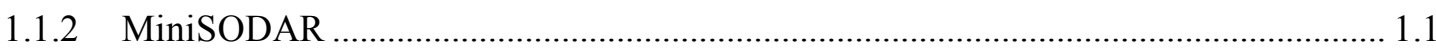

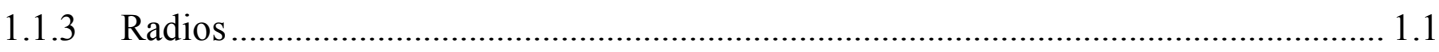

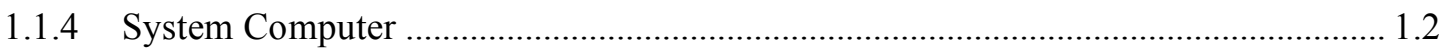

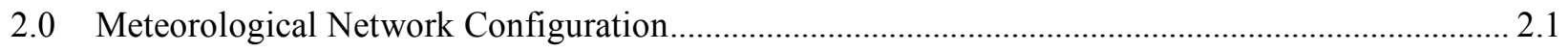

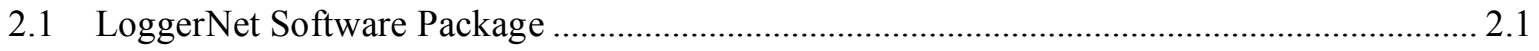

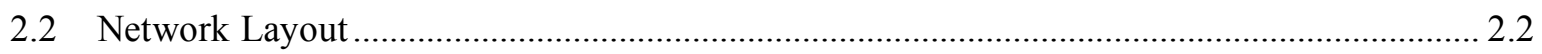

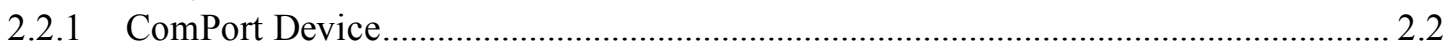

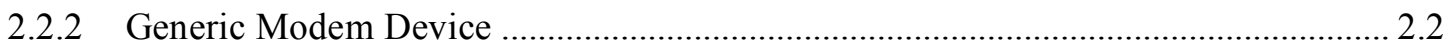

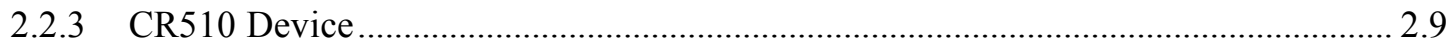

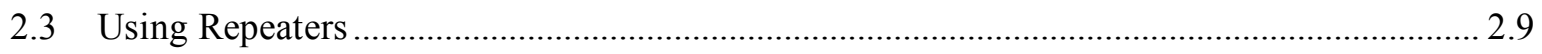

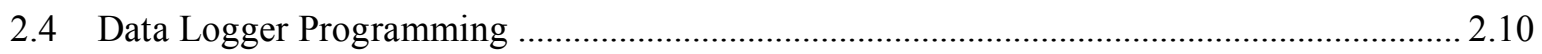

2.5 LoggerNet Backup and Transfer to Another Computer...................................................... 2.14

3.0 Radio Network, Transceiver Setup and Replacement …................................................................. 3.1

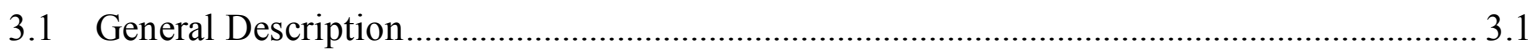

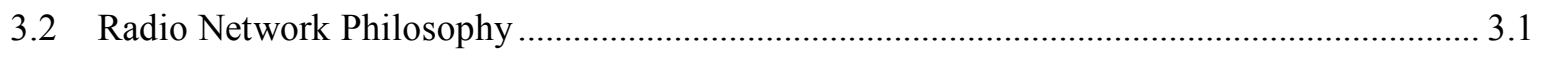

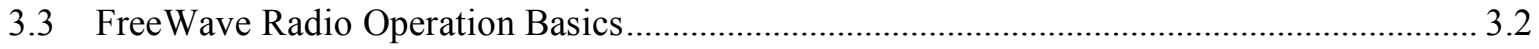

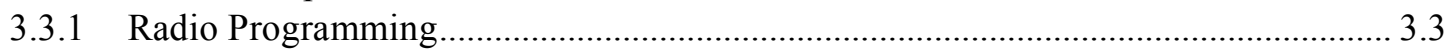

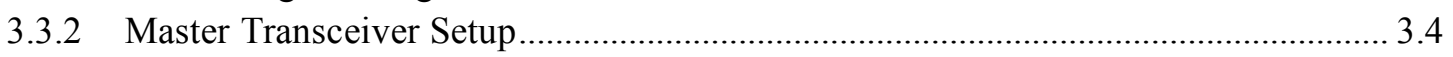

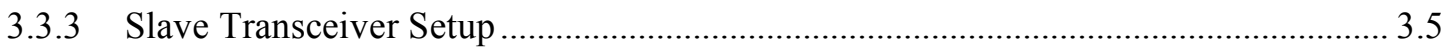

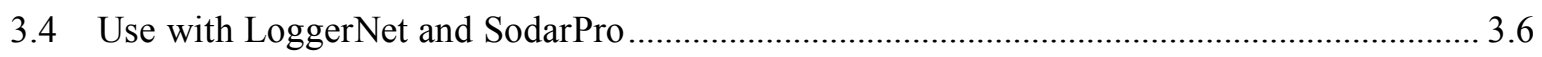

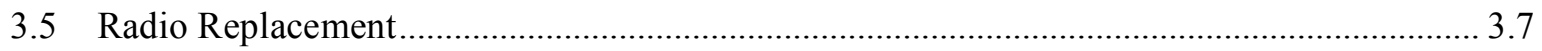

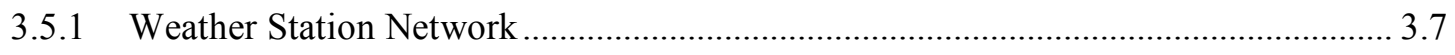

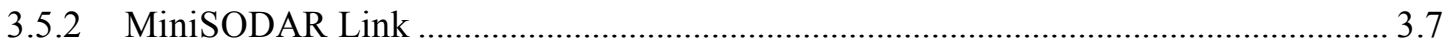

3.6 Current Transceiver Affiliation and Configuration.............................................................. 3.8

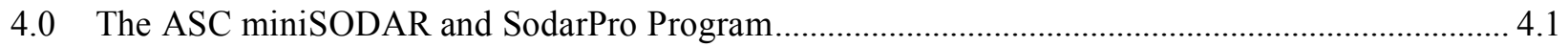

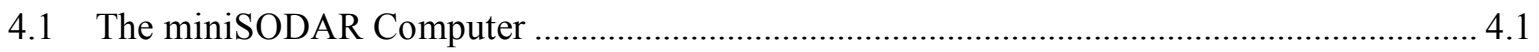

4.2 SodarPro Peculiarities .................................................................................................... 4.1

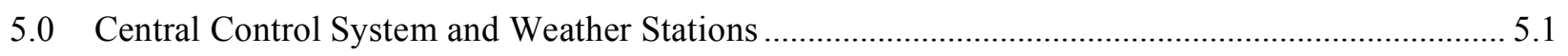

5.1 The Central Control System, Tools, and Spare Parts ............................................................ 5.1

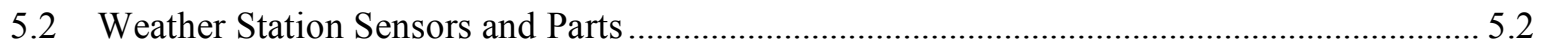

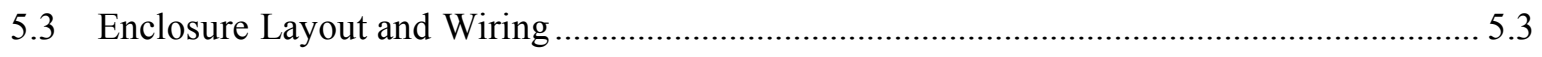

6.0 Operation of RDCDS Meteorological Component ....................................................................... 6.1

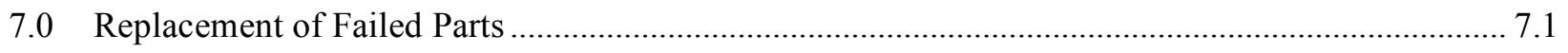




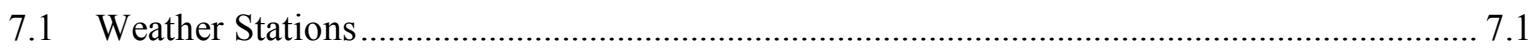

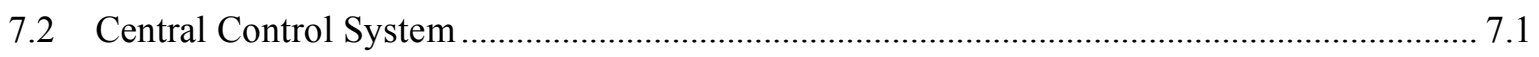

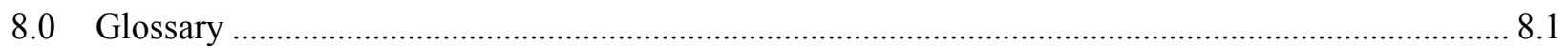

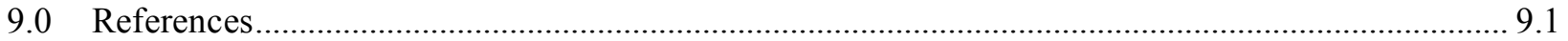

\section{Figures}

2.1. Screenshot of the Setup Utility Window with Highlighted ComPort, the Root Element of the

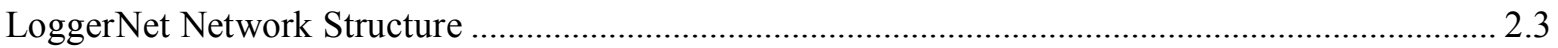

2.2. Screenshot of the Setup Utility Window with Highlighted Generic Modem Element and

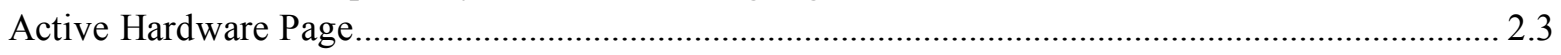

2.3. Screenshot of the Setup Utility Window with Highlighted Generic Modem Element and

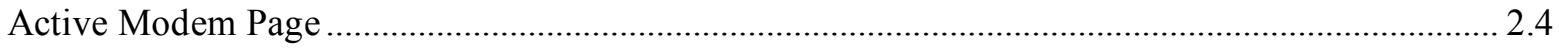

2.4. Screenshot of the Setup screen with Hardware Property Page of the CR510_1 Device. ................... 2.9

2.5. Short Cut Utility: Sensor Selection Window (second programming step)..................................... 2.11

2.6. Short Cut Utility: Output Signals and Processing Window for the Sensors Selected; 1-min

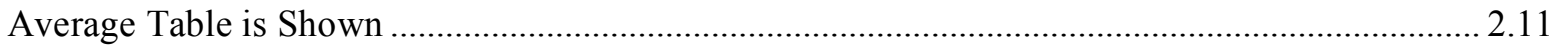

2.7. Short Cut Utility: Output Signals and Processing Window for the Sensors Selected; 5-min Average Table is Shown ........................................................................................................ 2.12

2.8. Short Cut Utility: Diagram of the Sensor Leads and Where Each Should be Connected on the Data Logger's Wiring Panel....................................................................................................... 2.12

2.9. Short Cut Utility: Wiring Text Window Which Provides a List of the Data Logger Channels

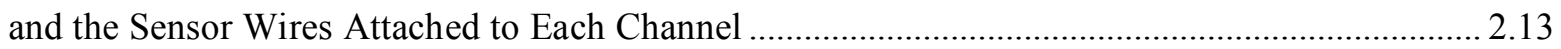

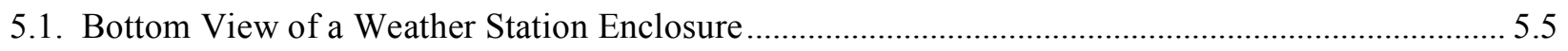

5.2. Inside View of the Enclosure. The large black rectangle is an internal battery. ................................ 5.6

5.3. Close View of the Data Logger Contact Panel and the Power Supply (battery charger) .................. 5.7

6.1. Laptop Screen with LoggerNet and SodarPro Windows. Purple labels have been added or clarity, and are not part of the display 


\section{Tables}

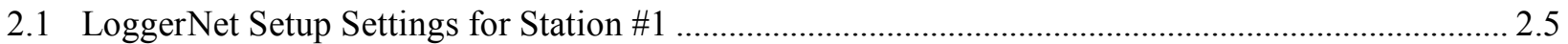

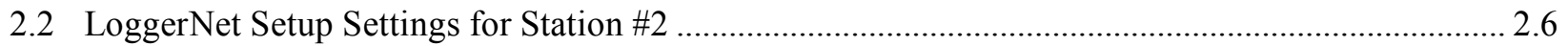

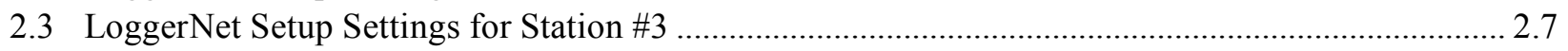

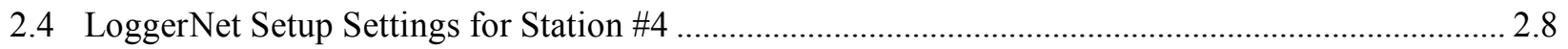

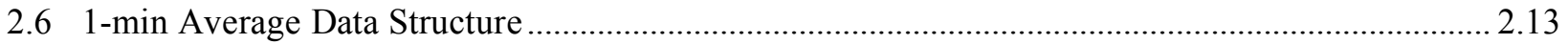

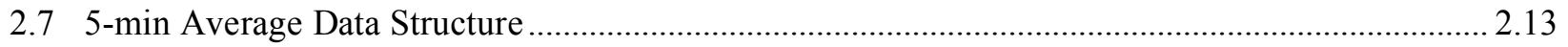

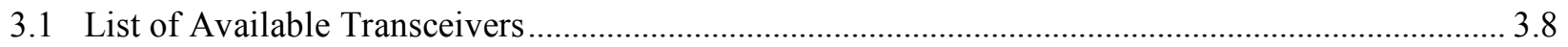

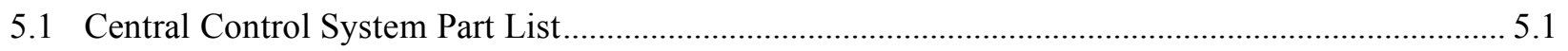

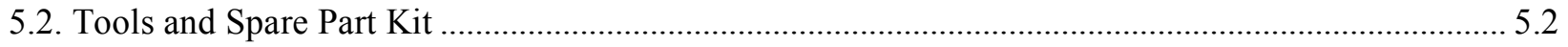

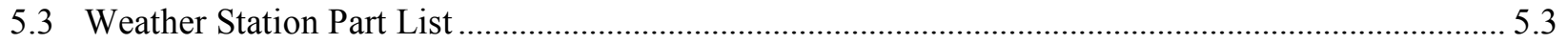

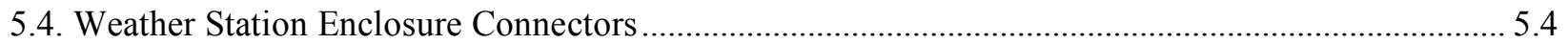

5.5 Wind Speed and Direction Sensor Connector Pinout and Wire Color Coding ................................. 5.5

5.6 Temperature Sensor Connector Pinout and Wire Color Coding ...................................................... 5.5

5.7 External Battery Connector Pinout and Wire Color Coding ............................................................. 5.6

5.8 Solar Panel Connector Pinout and Wire Color Coding ................................................................... 5.6 



\subsection{Introduction}

The Rapidly Deployable Chemical Defense System (RDCDS) has been developed to protect against indoor and outdoor releases of toxic industrial chemicals or chemical warfare agents. The system has been designed to be used in a wide range of venues and includes both ground-based and airborne components. The goal of the system is to provide real-time information to event organizers and first responders. This document describes the setup and use of the observational meteorological component of the RDCDS. Step-by-step installation instructions for the weather station and central control system can be found in the RDCDS Meteorological Component Quick Installation Guide (Berg and Pekour 2007).

\subsection{Description of system components}

\subsubsection{Weather Stations}

The RDCDS meteorological network consists of four identical weather stations. Each weather station consists of an anemometer, a thermometer, a data logger, a radio modem, and a folding aluminum tripod. The anemometer is a high performance propeller and vane anemometer that meets or exceeds requirements published by the U.S. Environmental Protection Agency (EPA 1987) and the U.S. Nuclear Regulatory Agency (NRC 2007). The anemometer is mounted on top of the tripod, approximately 10 feet above the ground. Ambient air temperature is measured using a thermistor mounted in a six-plate nonaspirated radiation shield. Data are stored, and average values of wind speed, wind direction, and temperature are computed using a Campbell Scientific CR510 Data Logger. The data logger and radio are mounted inside a small weatherproof enclosure. Power is supplied by a small internal battery or a large external battery, both of which are charged using a small solar panel. The data logger and radio are

configured for automatic start and continuous operation. Although one of the weather stations is intended to be used as a backup station or a radio repeater, it can be used as a fourth station if needed.

\subsubsection{MiniSODAR}

In addition to the weather stations, the RDCDS network includes an Atmospheric Systems Corporation miniSODAR (SOnic Detection and Ranging). This instrument is a Doppler acoustic sounder that measures profiles of wind speed and wind direction to an altitude of up to $200 \mathrm{~m}$ above the miniSODAR. The miniSODAR is controlled from the RDCDS command center using SodarPro software via a continuous radio link. While the continuous link requires an additional radio at the command center, it allows the on-site scientist to monitor the performance of the miniSODAR in real time. The miniSODAR is powered by AC power and/or through a bank of deep-cycle batteries. Details of the miniSODAR operation can be found in the manuals supplied by the vendor.

\subsubsection{Radios}

FreeWave Spread Spectrum Wireless Data Transceivers are used for the communications between the weather stations, the miniSODAR, and the network computer. These radios were selected for their long range (advertised as 60-mile line of sight). The meteorological network has been configured such that the radios can be used as a repeater for any of the weather stations. Two enclosed models are for use at the RDCDS command station. One enclosed radio is used for communication with the weather stations, and the other is for continuous communication with the miniSODAR. One additional enclosed radio is 
included as a backup for either of these radios. In addition, two omni directional antennas are provided for use at the command center. Further details about the radios can be found in the FreeWave Spread Spectrum Wireless Data Transceiver User Manual (FreeWave 2004).

\subsubsection{System Computer}

The meteorological component of the RDCDS network is controlled through a laptop computer at the RDCDS command center. This computer runs Campbell Scientific, Inc., LoggerNet software to facilitate communications with the weather stations, and Atmospheric Systems Corporation SodarPro software for control and communication with the miniSODAR. Data from the meteorological sensors can be transferred to the computer that is used for monitoring the chemical detection nodes. A backup laptop, which is configured exactly like the standard computer, is provided to protect against failure. 


\subsection{Meteorological Network Configuration}

The central control system computer serves as a data acquisition system to the whole RDCDS meteorological component. It uses the LoggerNet software package to control the meteorological network, and collect, average, and display recent data and network status. The miniSODAR is controlled via a serial interface over a radio link with the help of SodarPro program (see Section 1.0).

\subsection{LoggerNet Software Package}

LoggerNet, which was developed by Campbell Scientific, Inc., is a suite of software tools that contains several utilities. These utilities were used during the design and development of the network and are as follows:

1. LoggerNet server. Also known as the Toolbar, this utility performs all communications with devices on the network according to a user-defined schedule, and displays a tool bar on the computer screen to initiate and navigate all other applications.

2. Setup. This utility sets up and configures the network using simple "drag \& drop" interface, predefined set of data loggers, communication devices, and other "building blocks." This utility includes a wide range of communication/schedule/store options.

3. Connect. This utility checks out and debugs a connection to a remote data logger with capabilities to establish a connection, display current state and data flow from the data logger, as well as upload and compile a program on the data logger.

4. Status Monitor. This utility monitors current status of all network links, with tracing, logging, and debugging capabilities.

5. Short Cut. This utility creates, uploads, and compiles a program on a data logger. This utility includes a simple graphical interface, a wide choice of predefined sensors and data loggers, measurements, averages, etc.

6. RTMC Dev. The Real Time Monitor and Control Development utility creates a graphical display of collected data using pre-programmed features.

7. RTMC RT. The Real Time Monitor and Control Development Run-Time utility shows a graphical display prepared with the RTMC Dev.

Under normal circumstances, after all the equipment has been set up, the LoggerNet server is the only program necessary for the current application (e.g., it polls all remote stations, collects the data, keeps $\operatorname{logs}$, etc). The RTMC RT and Status Monitor are used to monitor the network, diagnose current conditions, and display meteorological information in several forms (i.e., current status, time history for 1, 12, and $48 \mathrm{~h}$ ). The Setup and Connect may be necessary during network setup or repair of a damaged or failed network. The Short Cut may be needed if a data logger program needs to be changed (e.g., a new type of a sensor and/or a new data logger is used). 


\subsection{Network Layout}

There are two layers in the concept of the RDCDS Meteorological Component network layout. The first is a "logical" layer, which is essentially a reflection of the information flows and controls; it consist of logical devices and links between them. The underlying network layer is a "hardware" layer, or radio network, which includes all physical devices and links (radio, serial, software, etc.) necessary to achieve the desired controls and information flows ("logical" layer).

The network design pursued two priorities (besides obvious ones, like reliability, ease of setup and operation): 1) minimum effort, and consequently, minimum down time, in case of any hardware failure; and 2) maximum flexibility in the radio network topology including ease of use of repeaters (weather stations and/or designated repeaters) without major reprogramming of the radios. In most cases, replacement of the failed remote station requires a visit to the remote site for hardware replacement, and in some cases, it also requires simple corrections in the LoggerNet Setup on the central control system computer; no calls on other sites are necessary. Flexibility in defining the radio network topology should facilitate deployment of the system in various locations because the physical network layout depends on local conditions. The operational decisions on specific network layout and repeater usage should be made at the time of deployment and testing.

The LoggerNet network ("logical" layer) has a simple star layout; in other words, each data logger is only aware of the central control system laptop (see Setup screenshot in Figure 2.1; the left-hand side of the screen shows the network layout and the logical devices and connections between them). Four CR510 data loggers (three remote weather stations and a spare) are connected to one "ComPort" of the central control system computer via four separate "Generic Modem" devices. Specific operational parameters (poll schedule, timings, routing, etc.) are stored in the Generic Modem property pages (see Figure 2.2 and Figure 2.3). The radio network is completely transparent to LoggerNet, and the radio network may have a different and more complicated topology than the LoggerNet network (tree, daisy chain, or combination). For example, LoggerNet is not aware of the use of radio repeaters.

\subsubsection{ComPort Device}

This logical device has one property page named "Hardware" (see Figure 2.1); it contains a Communication Enabled checkbox (which should be checked). The usual choice for "Comm Port Connection" is COM4. Most current laptops lack serial ports; therefore, a 4-port USB-to-serial adapter is provided. Typically, the ports on the adapter are mapped on communication devices COM4 through COM7. No Advanced features of ComPort device are used.

\subsubsection{Generic Modem Device}

The Generic Modem is a general-purpose communication device. It allows the use of modem-like AT-commands in "send-receive" dialog to control link initialization and setup. This device has two property pages: "Hardware" and "Modem" (Figure 2.2 and Figure 2.3). See Table 2.1-Table 2.4 for specific information on the settings of each station, which are stored in these property pages.

The "Modem" property page of the Generic Modem device contains an important command line in the "Dial Script" field. This command line is to be sent to the master radio transceiver to establish a radio link with a particular slave radio, (i.e., to the prescribed remote station and data logger). After the master 


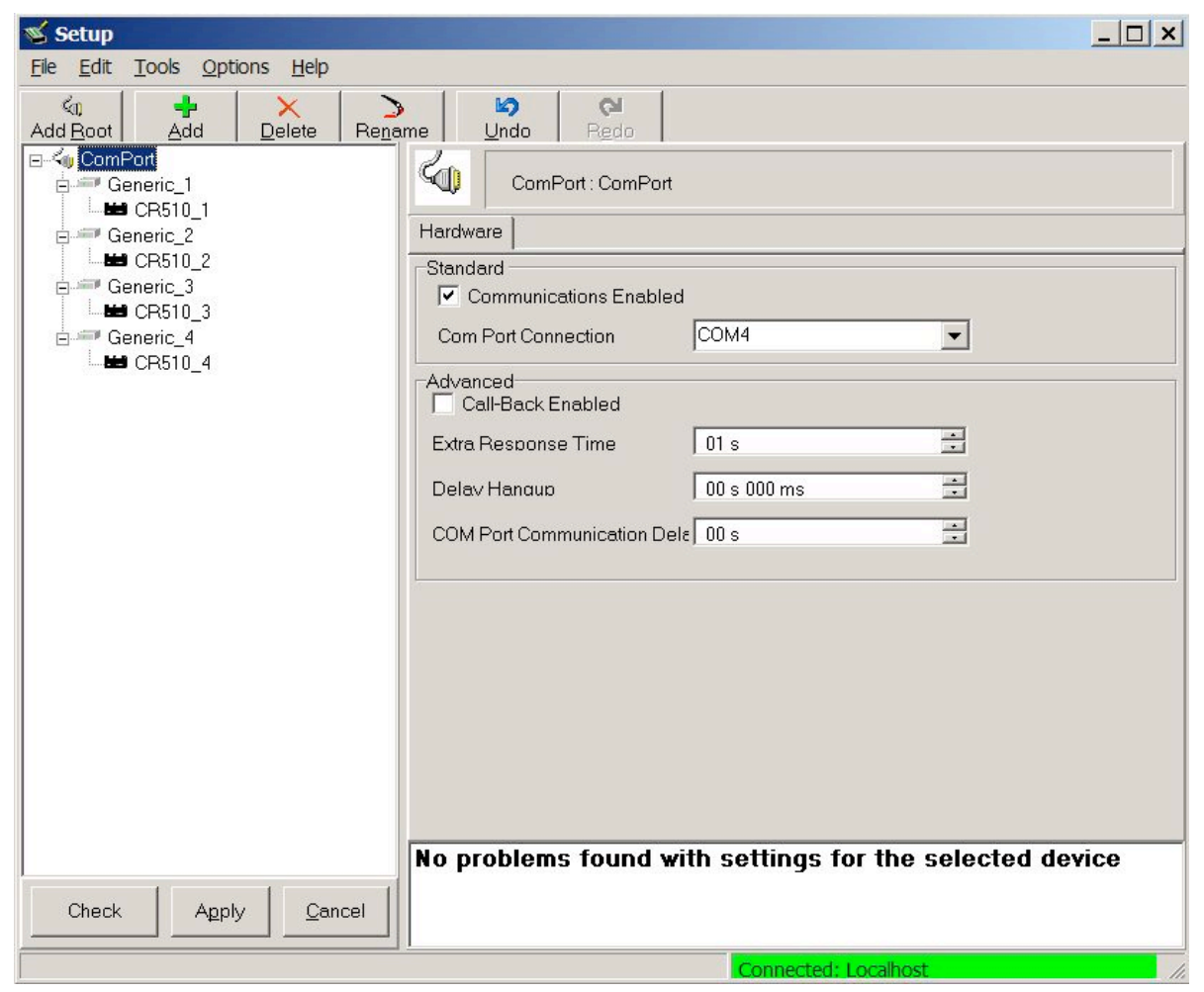

Figure 2.1. Screenshot of the Setup Utility Window with Highlighted ComPort, the Root Element of the LoggerNet Network Structure

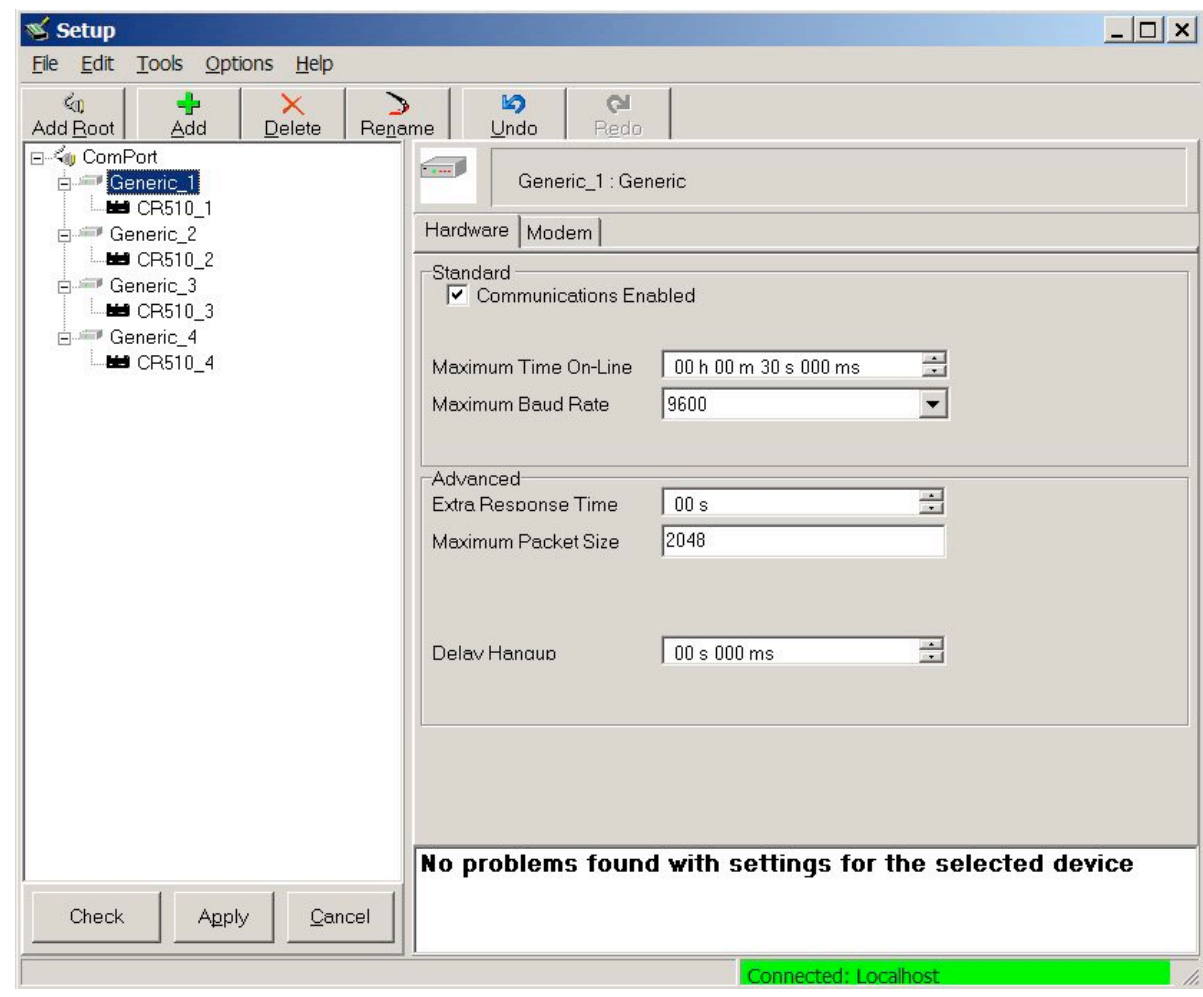

Figure 2.2. Screenshot of the Setup Utility Window with Highlighted Generic Modem Element and Active Hardware Page 


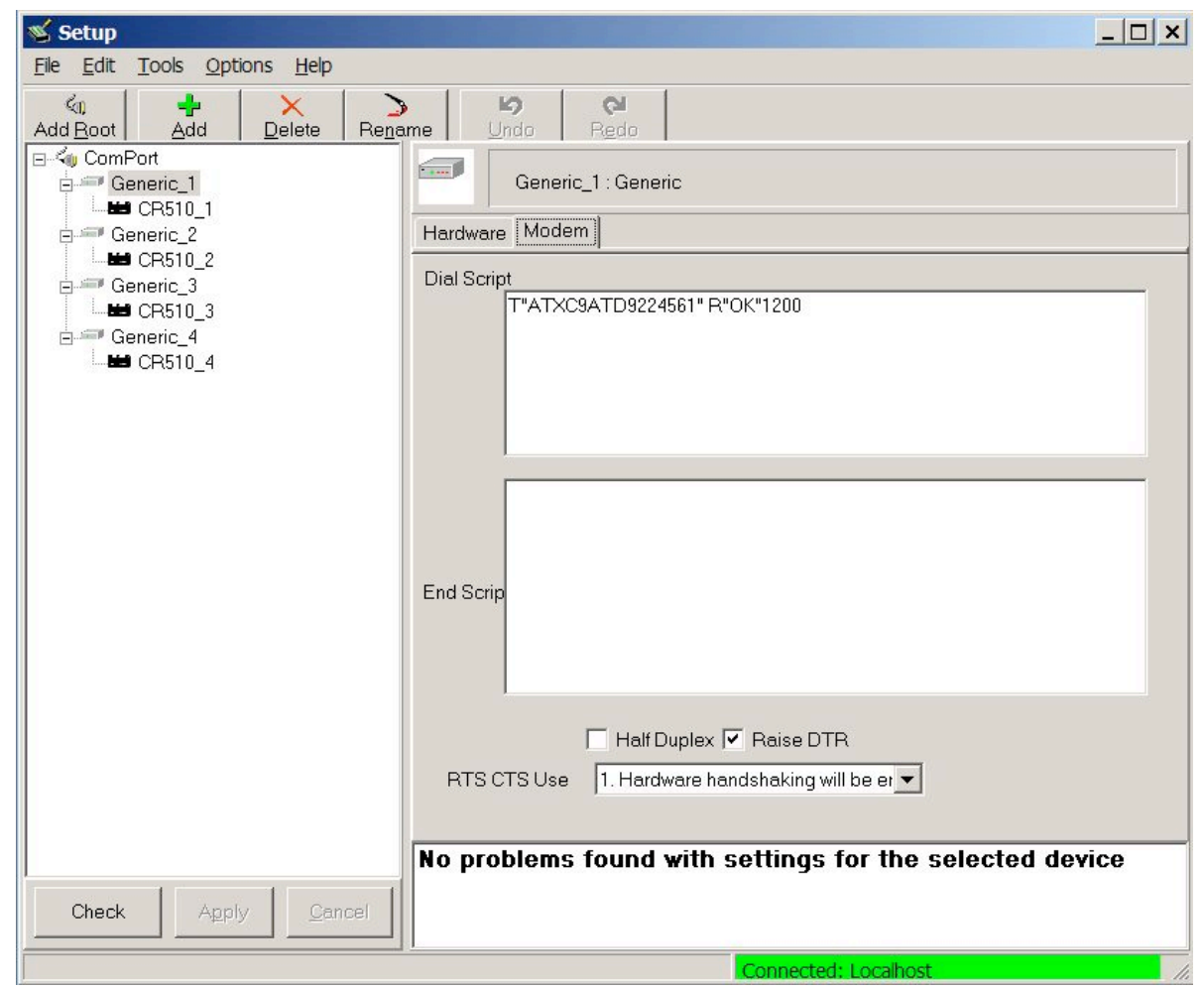

Figure 2.3. Screenshot of the Setup Utility Window with Highlighted Generic Modem Element and Active Modem Page

radio establishes a radio link, its operation becomes fully transparent to LoggerNet (just like a wired serial line). See also section "Radio Setup" and the section entitled, "Set Operation Mode" in the FreeWave Spread Spectrum Wireless Data Transceiver User Manual (FreeWave 2004). Actually, an aggregate of these "Dial Scripts" defines the topology of the radio network because each slave radio is connected physically to a specific data logger. In addition, the scripts contain routing information, which describes implicitly the necessary radio links to achieve a two-way connection with a particular remote station.

The calling mode for a particular station depends on the desired network configuration, polling sequence, etc. It should be defined during initial network setup and testing. In the current application, we use a combination of two FreeWave radio commands: 1) change Entry to Call (defines routing to use) and 2) call a specific transceiver. The calling command string contains ATXCXATD\#\#\#\#\#\# where ATXCX is request to change Entry to Call to line $\mathrm{x}$, and ATD\#\#\#\#\#\# is command to call slave transceiver with ID of \#\#\#\#\#\#\# using routing defined by the Entry to Call, in this case a repeater (if any) from field "Repeater1" of line $\mathrm{x}$ in the Master Call Book. This combination of commands allows for direct calls to a specific slave as well as routed calls though one repeater (see Section 3.2 for details of our convention on Call Books setup).

For example, the command line for the first station is T"ATXC9ATD9224561"R"OK"1200. This command line can be interpreted as "connect to the slave 9224561 using repeater described in line 9 of Master Call Book and wait for response OK for 1200 ms." By our convention and because line 9 has no repeater, the master radio will try to make a direct link with the slave 9224561 . This is installed at the first station and connected to data logger 1 (see Section 3.6 for current transceiver associations). 
Table 2.1. LoggerNet Setup Settings for Station \#1

\begin{tabular}{|c|c|c|}
\hline $\begin{array}{l}\text { Location in the LoggerNet Setup } \\
\text { (logical device } \rightarrow \text { property page) }\end{array}$ & Parameter Name & Value \\
\hline \multirow[t]{6}{*}{ Generic_1 $\rightarrow$ Hardware } & Communications Enabled & Checked \\
\hline & Maximum Time On-Line & $30 \mathrm{~s}$ \\
\hline & Maximum Baud Rate & 9600 \\
\hline & Extra Response Time & 0 (default, not used) \\
\hline & Maximum Packet Size & 2048 (not changed from default) \\
\hline & Delay Hangup & 0 (default, not used) \\
\hline \multirow[t]{5}{*}{ Generic $1 \rightarrow$ Modem } & Dial Script & T”ATXC9ATD9224561"R"OK”1200 \\
\hline & End Script & (empty, not used here) \\
\hline & Half Duplex & Not checked \\
\hline & Raise DTR & Checked \\
\hline & RTS CTS Use & 1. Hardware handshaking \\
\hline \multirow[t]{8}{*}{ CR510_1 $\rightarrow$ Hardware } & Communications Enabled & Checked \\
\hline & Maximum Time On-Line & $30 \mathrm{~s}$ \\
\hline & Maximum Baud Rate & 9600 \\
\hline & Extra Response Time & 0 (default, not used) \\
\hline & Maximum Packet Size & 2048 (not changed from default) \\
\hline & Security Code & 0 (default, not used) \\
\hline & Call-Back ID & 1 (just in case, not used) \\
\hline & Delay Hangup & 0 (default, not used) \\
\hline \multirow[t]{8}{*}{ CR510_1 $\rightarrow$ Schedule } & Scheduled Collection Enabled & Checked \\
\hline & Base Date & $1 / 1 / 1990$ \\
\hline & Base Time & 00:00:00 \\
\hline & Collection Interval & $5 \mathrm{~m} \mathrm{00s}$ \\
\hline & Primary Retrv. Interval & $20 \mathrm{~s}$ \\
\hline & Number of Primary Retries & 3 \\
\hline & Secondary Retry Interval & Not checked \\
\hline & Collect Ports and Flags & Checked \\
\hline \multirow[t]{8}{*}{ CR510_1 $\rightarrow$ FS Area 1} & Enabled for Collection & Checked \\
\hline & Output File Name & $\begin{array}{l}\text { C:ICampbellscilLoggerNet|CR510_1_fin } \\
\text { al_storage 1.dat }\end{array}$ \\
\hline & Use Default File Name & Not checked \\
\hline & File Output Option & Append to End of File \\
\hline & Output Format & ASCII, Comma Separated \\
\hline & $\begin{array}{l}\text { Collect Mode, Data Logged Since Last } \\
\text { Collection }\end{array}$ & Chosen \\
\hline & Collect All On First Collection & Checked \\
\hline & Collect Mode, Most Recently Logged Arrays & Not chosen \\
\hline \multirow[t]{8}{*}{ CR510_1 $\rightarrow$ FS Area 2} & Enabled for Collection & Checked \\
\hline & Output File Name & $\begin{array}{l}\text { C:ICampbellscilLoggerNet|CR510_1_fin } \\
\text { al_storage_2.dat }\end{array}$ \\
\hline & Use Default File Name & Not checked \\
\hline & File Output Option & Append to End of File \\
\hline & Output Format & ASCII, Comma Separated \\
\hline & $\begin{array}{l}\text { Collect Mode, Data Logged Since Last } \\
\text { Collection }\end{array}$ & Chosen \\
\hline & Collect All On First Collection & Checked \\
\hline & Collect Mode, Most Recently Logged Arrays & Not chosen \\
\hline \multirow[t]{6}{*}{ CR510_1 $\rightarrow$ Clock } & Time Zone Offset & $0 \mathrm{~h} 0 \mathrm{~m}$ \\
\hline & Automated Clock Check, Enabled & Checked \\
\hline & Automated Clock Check, Initial Date & $1 / 1 / 1990$ \\
\hline & Automated Clock Check, Initial Time & 00:00:00 \\
\hline & Automated Clock Check, Interval & $0 \mathrm{~d} 08 \mathrm{~h} 00 \mathrm{~m}$ \\
\hline & $\begin{array}{l}\text { Automated Clock Check, Allowed Clock } \\
\text { Deviation }\end{array}$ & $02 \mathrm{~s}$ \\
\hline CR510 $1 \rightarrow$ Program & Current Program & dl 1.dld \\
\hline
\end{tabular}


Table 2.2. LoggerNet Setup Settings for Station \#2

\begin{tabular}{|c|c|c|}
\hline $\begin{array}{l}\text { Location in the LoggerNet Setup } \\
\text { (logical device } \rightarrow \text { property page) }\end{array}$ & Parameter Name & Value \\
\hline \multirow[t]{6}{*}{ Generic_2 $\rightarrow$ Hardware } & Communications Enabled & checked \\
\hline & Maximum Time On-Line & $30 \mathrm{~s}$ \\
\hline & Maximum Baud Rate & 9600 \\
\hline & Extra Response Time & 0 (default, not used) \\
\hline & Maximum Packet Size & 2048 (not changed from default) \\
\hline & Delay Hangup & 0 (default, not used) \\
\hline \multirow[t]{5}{*}{ Generic_2 $\rightarrow$ Modem } & Dial Script & T”ATXC9ATD9224561"R"OK"1200 \\
\hline & End Script & (empty, not used here) \\
\hline & Half Duplex & Not checked \\
\hline & Raise DTR & Checked \\
\hline & RTS CTS Use & 1. Hardware handshaking \\
\hline \multirow[t]{8}{*}{ CR510_2 $\rightarrow$ Hardware } & Communications Enabled & checked \\
\hline & Maximum Time On-Line & $30 \mathrm{~s}$ \\
\hline & Maximum Baud Rate & 9600 \\
\hline & Extra Response Time & 0 (default, not used) \\
\hline & Maximum Packet Size & 2048 (not changed from default) \\
\hline & Security Code & 0 (default, not used) \\
\hline & Call-Back ID & 1 (just in case, not used) \\
\hline & Delay Hangup & 0 (default, not used) \\
\hline \multirow[t]{8}{*}{ CR510_2 $\rightarrow$ Schedule } & Scheduled Collection Enabled & Checked \\
\hline & Base Date & $1 / 1 / 1990$ \\
\hline & Base Time & 00:01:00 (1 min after station \#1) \\
\hline & Collection Interval & $5 \mathrm{~m} \mathrm{00s}$ \\
\hline & Primary Retrv. Interval & $20 \mathrm{~s}$ \\
\hline & Number of Primary Retries & 3 \\
\hline & Secondary Retry Interval & Not checked \\
\hline & Collect Ports and Flags & Checked \\
\hline \multirow[t]{8}{*}{ CR510_2 $\rightarrow$ FS Area 1} & Enabled for Collection & Checked \\
\hline & Output File Name & $\begin{array}{l}\text { C:ICampbellsci } \backslash \text { LoggerNet } \backslash \text { CR510_2_fin } \\
\text { al_storage_1.dat }\end{array}$ \\
\hline & Use Default File Name & Not checked \\
\hline & File Output Option & Append to End of File \\
\hline & Output Format & ASCII, Comma Separated \\
\hline & $\begin{array}{l}\text { Collect Mode, Data Logged Since Last } \\
\text { Collection }\end{array}$ & Chosen \\
\hline & Collect All On First Collection & Checked \\
\hline & Collect Mode, Most Recently Logged Arrays & Not chosen \\
\hline \multirow[t]{8}{*}{ CR510_2 $\rightarrow$ FS Area 2} & Enabled for Collection & Checked \\
\hline & Output File Name & $\begin{array}{l}\text { C:ICampbellsci } \backslash \text { LoggerNet } \backslash \text { CR510_2_fin } \\
\text { al_storage_2.dat }\end{array}$ \\
\hline & Use Default File Name & Not checked \\
\hline & File Output Option & Append to End of File \\
\hline & Output Format & ASCII, Comma Separated \\
\hline & $\begin{array}{l}\text { Collect Mode, Data Logged Since Last } \\
\text { Collection }\end{array}$ & Chosen \\
\hline & Collect All On First Collection & Checked \\
\hline & Collect Mode, Most Recently Logged Arrays & Not chosen \\
\hline \multirow[t]{6}{*}{ CR510_2 $\rightarrow$ Clock } & Time Zone Offset & $0 \mathrm{~h} 0 \mathrm{~m}$ \\
\hline & Automated Clock Check, Enabled & Checked \\
\hline & Automated Clock Check, Initial Date & $1 / 1 / 1990$ \\
\hline & Automated Clock Check, Initial Time & $00: 00: 00$ \\
\hline & Automated Clock Check, Interval & $0 \mathrm{~d} 08 \mathrm{~h} 00 \mathrm{~m}$ \\
\hline & $\begin{array}{l}\text { Automated Clock Check, Allowed Clock } \\
\text { Deviation }\end{array}$ & $02 \mathrm{~s}$ \\
\hline CR510_2 $\rightarrow$ Program & Current Program & dl_2.dld \\
\hline
\end{tabular}


Table 2.3. LoggerNet Setup Settings for Station \#3.

\begin{tabular}{|c|c|c|}
\hline $\begin{array}{l}\text { Location in the LoggerNet Setup } \\
\text { (logical device } \rightarrow \text { property page) }\end{array}$ & Parameter Name & Value \\
\hline \multirow[t]{6}{*}{ Generic_3 $\rightarrow$ Hardware } & בCommunications Enabled & Checked \\
\hline & Maximum Time On-Line & $30 \mathrm{~s}$ \\
\hline & Maximum Baud Rate & 9600 \\
\hline & Extra Response Time & 0 (default, not used) \\
\hline & Maximum Packet Size & 2048 (not changed from default) \\
\hline & Delay Hangup & 0 (default, not used) \\
\hline \multirow[t]{5}{*}{ Generic_3 $\rightarrow$ Modem } & Dial Script & T"ATXC9ATD9224561"R"OK"1200 \\
\hline & End Script & (empty, not used here) \\
\hline & Half Duplex & Not checked \\
\hline & Raise DTR & Checked \\
\hline & RTS CTS Use & 1. Hardware handshaking \\
\hline \multirow[t]{8}{*}{ CR510 $3 \rightarrow$ Hardware } & Communications Enabled & Checked \\
\hline & Maximum Time On-Line & $30 \mathrm{~s}$ \\
\hline & Maximum Baud Rate & 9600 \\
\hline & Extra Response Time & 0 (default, not used) \\
\hline & Maximum Packet Size & 2048 (not changed from default) \\
\hline & Security Code & 0 (default, not used) \\
\hline & Call-Back ID & 1 (just in case, not used) \\
\hline & Delay Hangup & 0 (default, not used) \\
\hline \multirow[t]{8}{*}{ CR510_3 $\rightarrow$ Schedule } & Scheduled Collection Enabled & Checked \\
\hline & Base Date & $1 / 1 / 1990$ \\
\hline & Base Time & 00:02:00 (2 min after station \#1) \\
\hline & Collection Interval & $5 \mathrm{~m} 00 \mathrm{~s}$ \\
\hline & Primary Retrv. Interval & $20 \mathrm{~s}$ \\
\hline & Number of Primary Retries & 3 \\
\hline & Secondary Retry Interval & Not checked \\
\hline & Collect Ports and Flags & Checked \\
\hline \multirow[t]{8}{*}{ CR510_3 $\rightarrow$ FS Area 1} & Enabled for Collection & Checked \\
\hline & Output File Name & $\begin{array}{l}\text { C: } \backslash \text { Campbellsci } \backslash \text { LoggerNet } \backslash \text { CR510_3_fin } \\
\text { al_storage_1.dat }\end{array}$ \\
\hline & Use Default File Name & Not checked \\
\hline & File Output Option & Append to End of File \\
\hline & Output Format & ASCII, Comma Separated \\
\hline & $\begin{array}{l}\text { Collect Mode, Data Logged Since Last } \\
\text { Collection }\end{array}$ & Chosen \\
\hline & Collect All On First Collection & Checked \\
\hline & Collect Mode, Most Recently Logged Arrays & Not chosen \\
\hline \multirow[t]{8}{*}{ CR510 3 $\rightarrow$ FS Area 2} & Enabled for Collection & Checked \\
\hline & Output File Name & $\begin{array}{l}\text { C:ICampbellscilLoggerNet } \backslash \text { CR510_3_fin } \\
\text { al_storage_2.dat }\end{array}$ \\
\hline & Use Default File Name & Not checked \\
\hline & File Output Option & Append to End of File \\
\hline & Output Format & ASCII, Comma Separated \\
\hline & $\begin{array}{l}\text { Collect Mode, Data Logged Since Last } \\
\text { Collection }\end{array}$ & Chosen \\
\hline & Collect All On First Collection & Checked \\
\hline & Collect Mode, Most Recently Logged Arrays & Not chosen \\
\hline \multirow[t]{6}{*}{ CR510_3 $\rightarrow$ Clock } & Time Zone Offset & $0 \mathrm{~h} 0 \mathrm{~m}$ \\
\hline & Automated Clock Check, Enabled & Checked \\
\hline & Automated Clock Check, Initial Date & $1 / 1 / 1990$ \\
\hline & Automated Clock Check, Initial Time & 00:00:00 \\
\hline & Automated Clock Check, Interval & $0 \mathrm{~d} 08 \mathrm{~h} 00 \mathrm{~m}$ \\
\hline & $\begin{array}{l}\text { Automated Clock Check, Allowed Clock } \\
\text { Deviation }\end{array}$ & $02 \mathrm{~s}$ \\
\hline CR510 3 $\rightarrow$ Program & Current Program & dl_3.dld \\
\hline
\end{tabular}


Table 2.4. LoggerNet Setup Settings for Station \#4

\begin{tabular}{|c|c|c|}
\hline $\begin{array}{l}\text { Location in the LoggerNet Setup } \\
\text { (logical device } \rightarrow \text { property page) }\end{array}$ & Parameter Name & Value \\
\hline \multirow[t]{6}{*}{ Generic_4 $\rightarrow$ Hardware } & Communications Enabled & Checked \\
\hline & Maximum Time On-Line & $30 \mathrm{~s}$ \\
\hline & Maximum Baud Rate & 9600 \\
\hline & Extra Response Time & 0 (default, not used) \\
\hline & Maximum Packet Size & 2048 (not changed from default) \\
\hline & Delay Hangup & 0 (default, not used) \\
\hline \multirow[t]{5}{*}{ Generic_4 $\rightarrow$ Modem } & Dial Script & T”ATXC9ATD9224561"R"OK"1200 \\
\hline & End Script & (empty, not used here) \\
\hline & Half Duplex & Not checked \\
\hline & Raise DTR & Checked \\
\hline & RTS CTS Use & 1. Hardware handshaking \\
\hline \multirow[t]{8}{*}{ CR510_4 $\rightarrow$ Hardware } & Communications Enabled & checked \\
\hline & Maximum Time On-Line & $30 \mathrm{~s}$ \\
\hline & Maximum Baud Rate & 9600 \\
\hline & Extra Response Time & 0 (default, not used) \\
\hline & Maximum Packet Size & 2048 (not changed from default) \\
\hline & Security Code & 0 (default, not used) \\
\hline & Call-Back ID & 1 (just in case, not used) \\
\hline & Delay Hangup & 0 (default, not used) \\
\hline \multirow[t]{8}{*}{ CR510_4 $\rightarrow$ Schedule } & Scheduled Collection Enabled & Checked \\
\hline & Base Date & $1 / 1 / 1990$ \\
\hline & Base Time & 00:03:00 (3 min after station \#1) \\
\hline & Collection Interval & $5 \mathrm{~m} \mathrm{00s}$ \\
\hline & Primary Retrv. Interval & $20 \mathrm{~s}$ \\
\hline & Number of Primary Retries & 3 \\
\hline & Secondary Retry Interval & Not checked \\
\hline & Collect Ports and Flags & Checked \\
\hline \multirow[t]{8}{*}{ CR510_4 $\rightarrow$ FS Area 1} & Enabled for Collection & Checked \\
\hline & Output File Name & $\begin{array}{l}\text { C:ICampbellsci } \backslash \text { LoggerNet } \backslash \text { CR510_4_fin } \\
\text { al_storage_1.dat }\end{array}$ \\
\hline & Use Default File Name & Not checked \\
\hline & File Output Option & Append to End of File \\
\hline & Output Format & ASCII, Comma Separated \\
\hline & $\begin{array}{l}\text { Collect Mode, Data Logged Since Last } \\
\text { Collection }\end{array}$ & Chosen \\
\hline & Collect All On First Collection & Checked \\
\hline & Collect Mode, Most Recently Logged Arrays & Not chosen \\
\hline \multirow[t]{8}{*}{ CR510 $4 \rightarrow$ FS Area 2} & Enabled for Collection & Checked \\
\hline & Output File Name & $\begin{array}{l}\text { C:ICampbellsci } \backslash \text { LoggerNet } \backslash \text { CR510_4_fin } \\
\text { al_storage_2.dat }\end{array}$ \\
\hline & Use Default File Name & Not checked \\
\hline & File Output Option & Append to End of File \\
\hline & Output Format & ASCII, Comma Separated \\
\hline & $\begin{array}{l}\text { Collect Mode, Data Logged Since Last } \\
\text { Collection }\end{array}$ & Chosen \\
\hline & Collect All On First Collection & Checked \\
\hline & Collect Mode, Most Recently Logged Arrays & Not chosen \\
\hline \multirow[t]{6}{*}{ CR510_4 $\rightarrow$ Clock } & Time Zone Offset & $0 \mathrm{~h} 0 \mathrm{~m}$ \\
\hline & Automated Clock Check, Enabled & Checked \\
\hline & Automated Clock Check, Initial Date & $1 / 1 / 1990$ \\
\hline & Automated Clock Check, Initial Time & $00: 00: 00$ \\
\hline & Automated Clock Check, Interval & $0 \mathrm{~d} 08 \mathrm{~h} 00 \mathrm{~m}$ \\
\hline & $\begin{array}{l}\text { Automated Clock Check, Allowed Clock } \\
\text { Deviation }\end{array}$ & $02 \mathrm{~s}$ \\
\hline CR510_4 $\rightarrow$ Program & Current Program & dl_4.dld \\
\hline
\end{tabular}


In the case of radio network changes (replacement of a failed radio, use of repeater(s), etc.), the Dial Script field on the Modem property page of the Generic_< $>$ $>$ device needs to be modified. FreeWave radios allow other calling modes (e.g., call via long chain of repeaters), but these modes require a different master Call Book setup. Detailed discussion of these capabilities falls beyond the scope of this document.

\subsubsection{CR510 Device}

The CR510 is a predefined logical device to control Campbell Scientific CR510 data loggers. It sets communication parameters, user-defined retrieval timings, data logger clock settings, data file names, storage locations, and various options on six property pages (see Figure 2.4 and Table 2.1-Table 2.4). The CR510 device also associates the data logger program with the data logger and contains controls to upload and compile the program on the data logger.

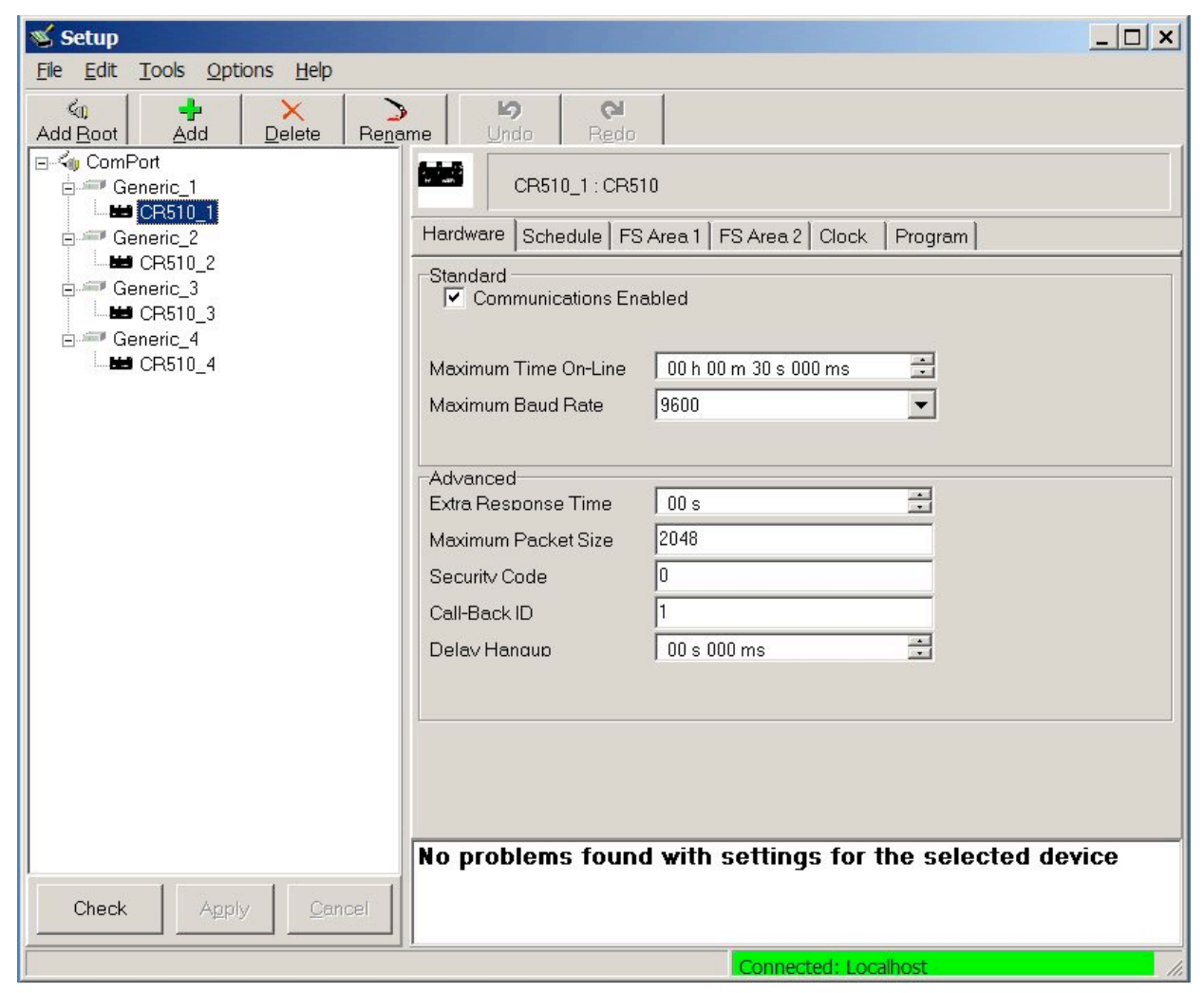

Figure 2.4. Screenshot of the Setup screen with Hardware Property Page of the CR510_1 Device.

\subsection{Using Repeaters}

Any slave transceiver listed in the master Call Book can be used as a repeater. No radio reconfiguration is necessary; all modifications are to be made in the LoggerNet Setup utility. This functionality is based on our convention to configure all of the radios in the network. The master radio Call Book contains the IDs of all radios as possible repeaters, and all slave Call Books contain IDs of all radios as possible masters (see also Section 1.0 on radio setup details). This arrangement allows us to choose or change a repeater setup in the software on the central control system computer. This is because 
the calling command line (stored in Dial Script value on the Modem property page of Generic_< $>$ device) defines the line of the master Call Book that contains the necessary repeater.

For example, if communication with station \#1 must go through station \#2 as a repeater, then the calling command line for station \#1 (currently T"ATXC9ATD9224561"R"OK"1200) needs to be changed to T"ATXC2ATD9224561"R"OK"1200. The ATXC2 stanza means that the master radio will try to connect with the slave radio ID 224516 (station \#1 transceiver) using a repeater listed in line 2 of the master Call Book, which is transceiver ID 921-8092 installed at station \#2 per our current assignment and our convention (see details on transceiver assignments and current Call Book contents in Section 1.0). The advanced use of repeaters falls beyond the scope of this document. For further details, see the section entitled, "Programming Point-to-Point Extended Call Book to Use Three or Four Repeaters" in the FreeWave Spread Spectrum Wireless Data Transceiver User Manual (FreeWave 2004)

\subsection{Data Logger Programming}

There are many ways to create a program for CR510 data loggers. We found that the Short Cut utility fully met our needs, and the current data logger program was created with the help of Short Cut. The same program is used on all remote stations with one site-specific modification: when a data logger was programmed for the first time, the data logger ID field in the "Settings $\rightarrow$ Data logger ID" menu of Short Cut was set to be equal to the station number. For all subsequent data logger reprogramming, this parameter should be 0 (default means no change to the data logger ID). Figure 2.5 presents screenshots of the Short Cut window at different steps of program creation. The data logger type is chosen on the first step ("New/Open" window, not shown on the screenshots). The next step, the "Sensor" window, allows selection of specific sensors from pre-defined lists (current selection is the Default, Temperature sensor 107, Wind sensor 05305, Battery voltage). The choice of information to collect is made on the "Outputs" window along with sampling rate, acquisition period, averaging, and storage assignment. Output information was divided into two output tables: table 101 and table 102 (Figures 2.6 and 2.7). Table 101 contains 1-min averages of wind speed and direction, air temperature, battery voltage, as well as time stamp and station ID (Figure 2.5, Table 2.5). Table 102 contains 5-min statistics. It contains scalar average and standard deviation of wind speed, vector average of wind speed and direction, standard deviation of wind direction, average and standard deviations of air temperature, and the average and minimum battery voltage (Figure 2.5 and Table 2.6). The output data are written into a file defined in LoggerNet Setup "CR510_2 $\rightarrow$ FS Area $1 \rightarrow$ Output File Name." In addition, Short Cut provides examples of the wiring that is appropriate for the chosen data logger and sensors in the form of text and diagrams (Figure 2.5 through Figure 2.9). 


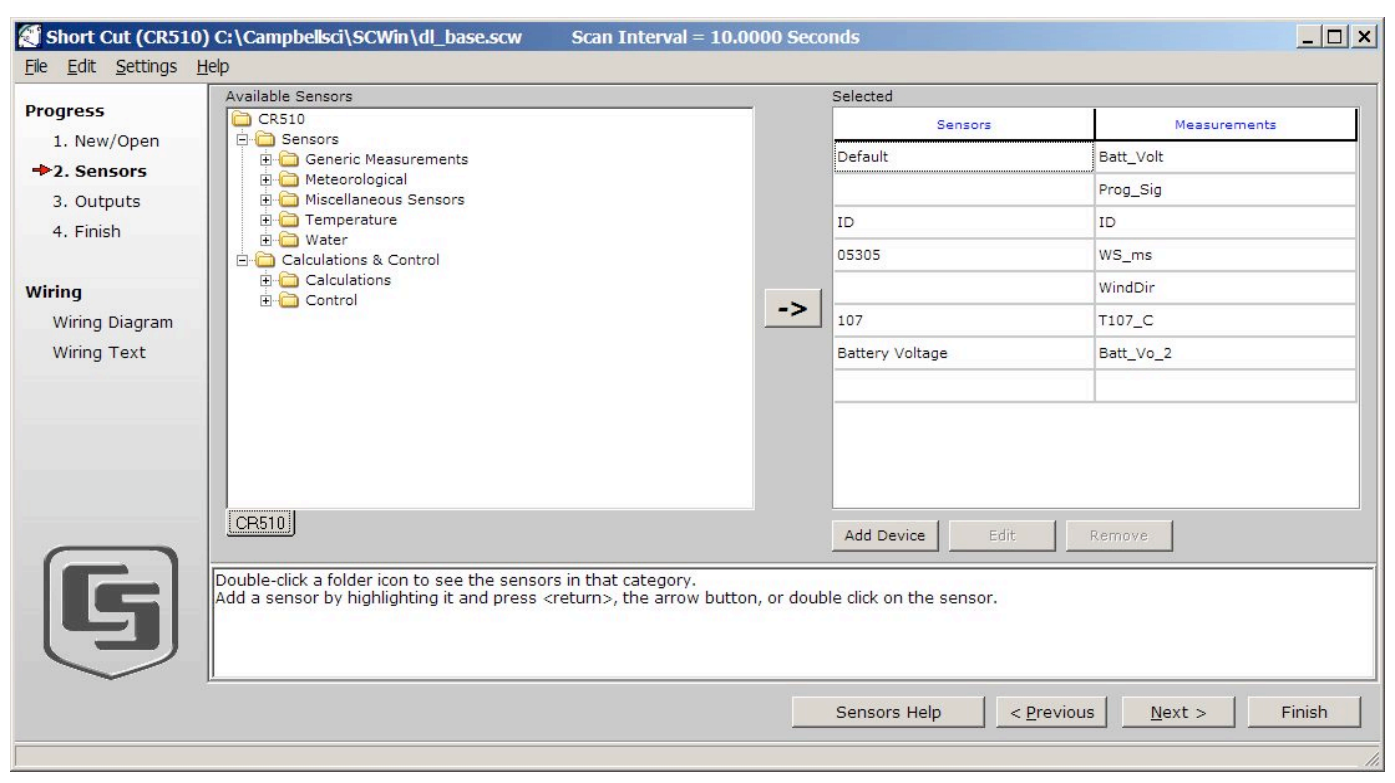

Figure 2.5. Short Cut Utility: Sensor Selection Window (second programming step).

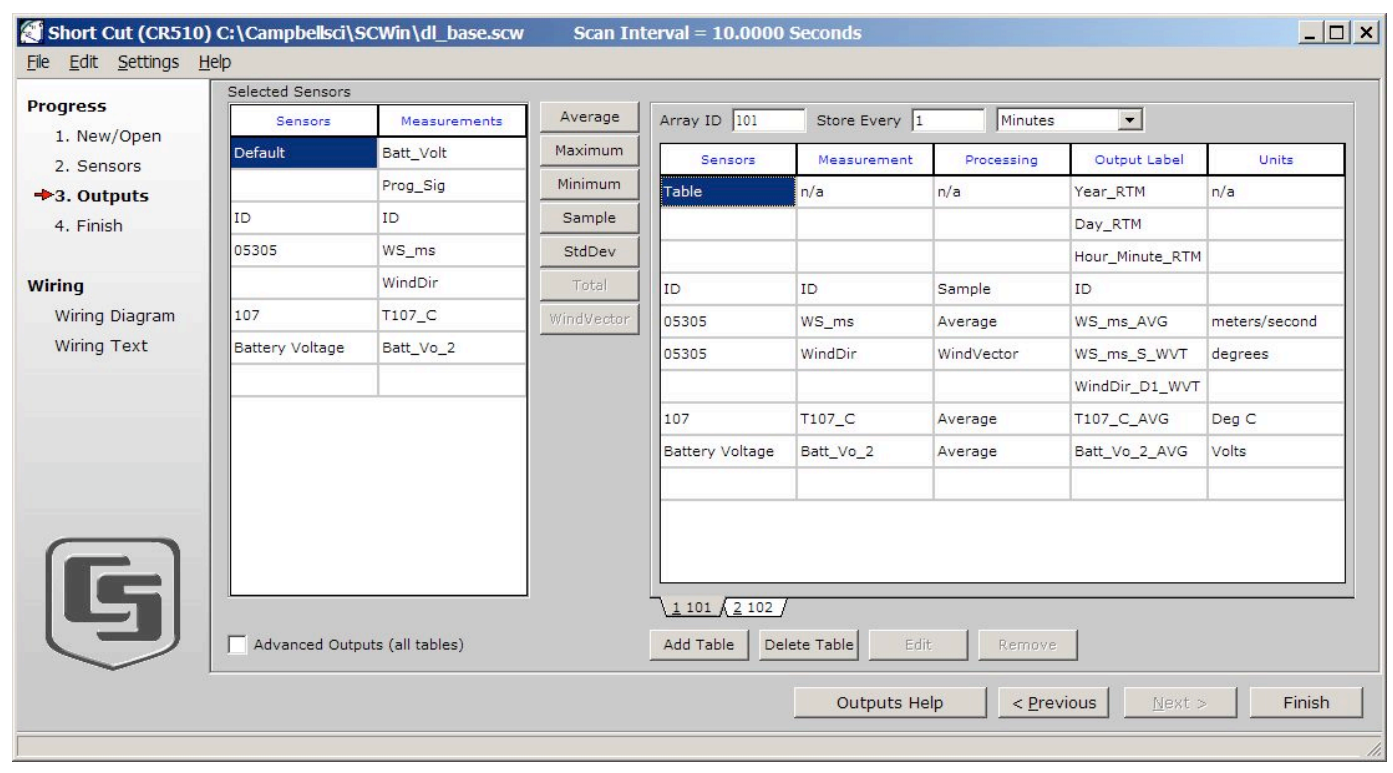

Figure 2.6. Short Cut Utility: Output Signals and Processing Window for the Sensors Selected; 1-min Average Table is Shown 


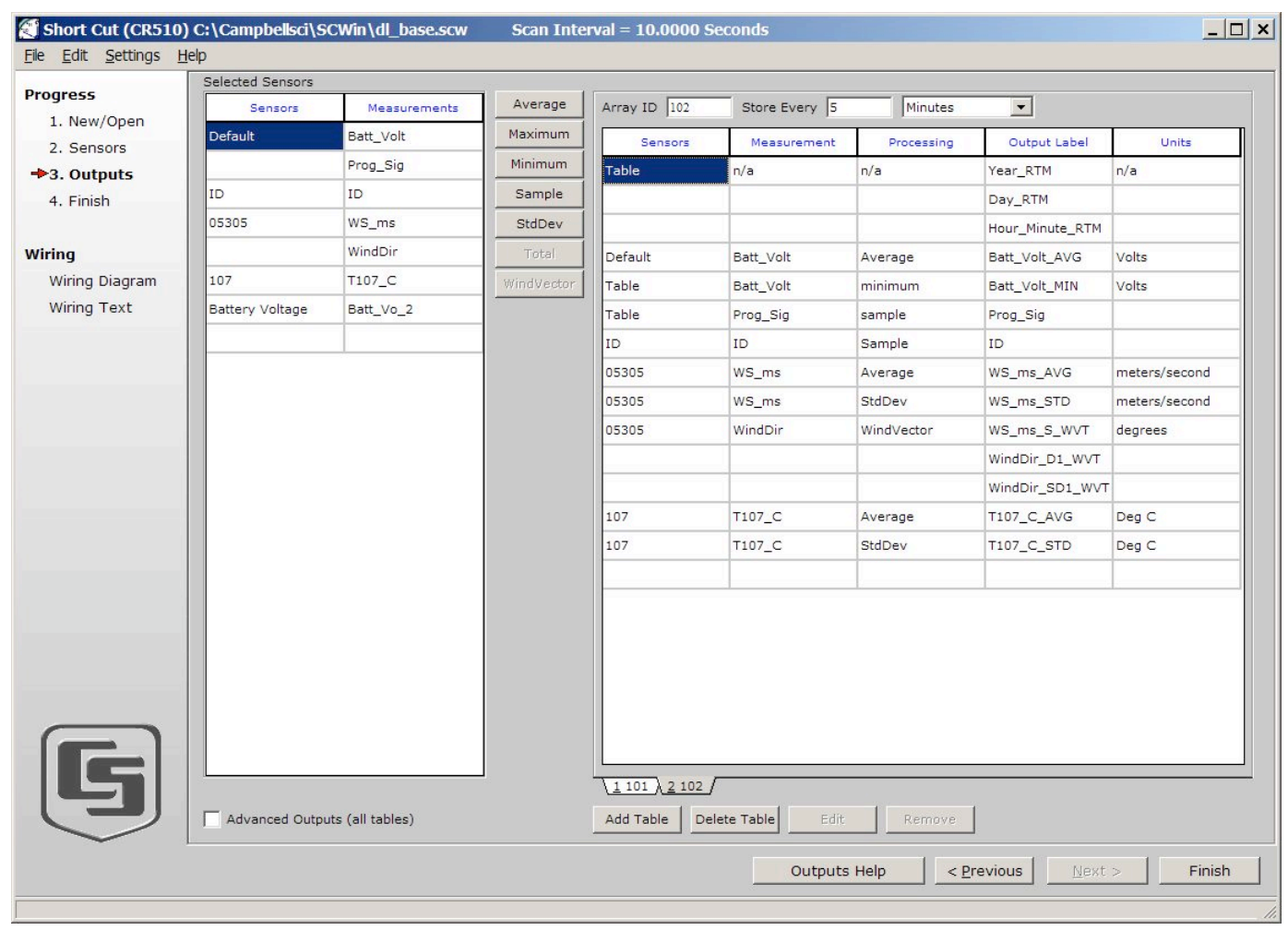

Figure 2.7. Short Cut Utility: Output Signals and Processing Window for the Sensors Selected; 5-min Average Table is Shown

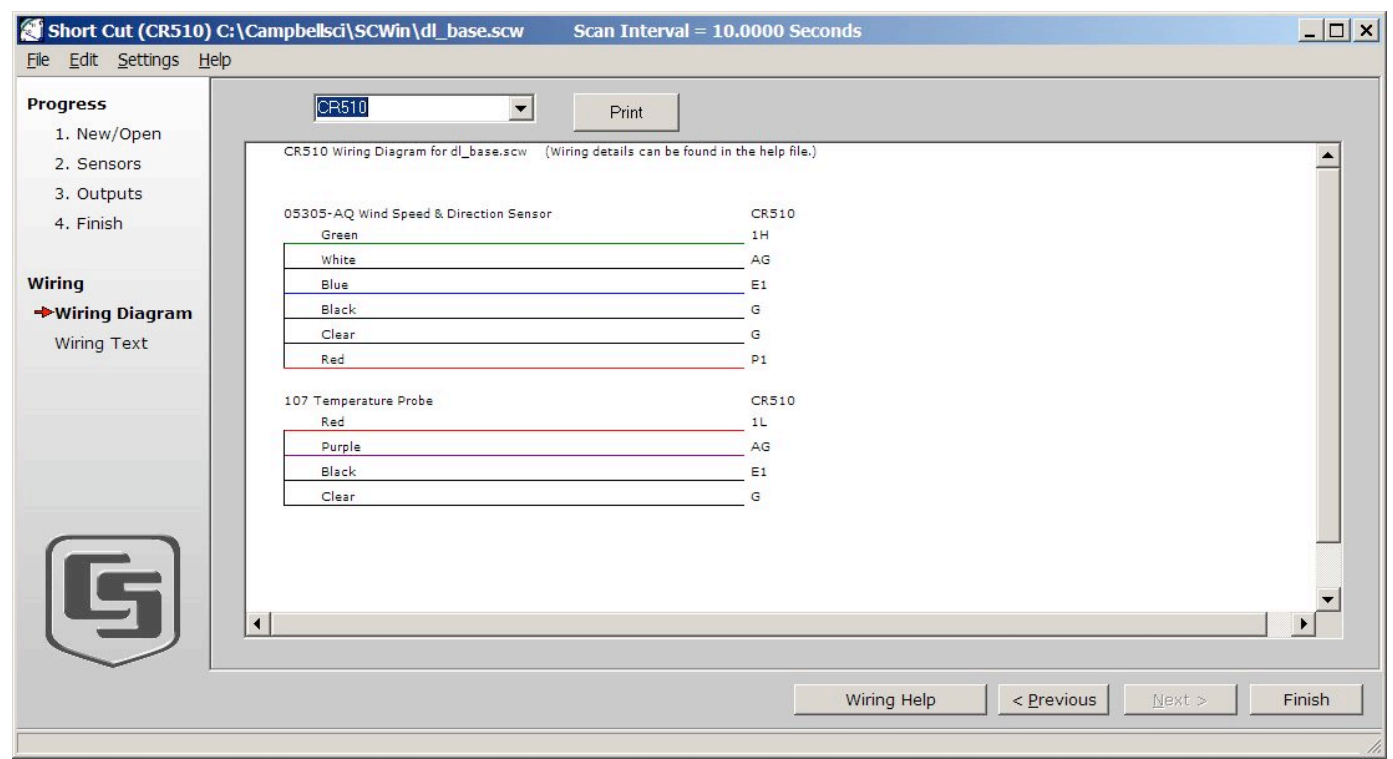

Figure 2.8. Short Cut Utility: Diagram of the Sensor Leads and Where Each Should be Connected on the Data Logger's Wiring Panel 


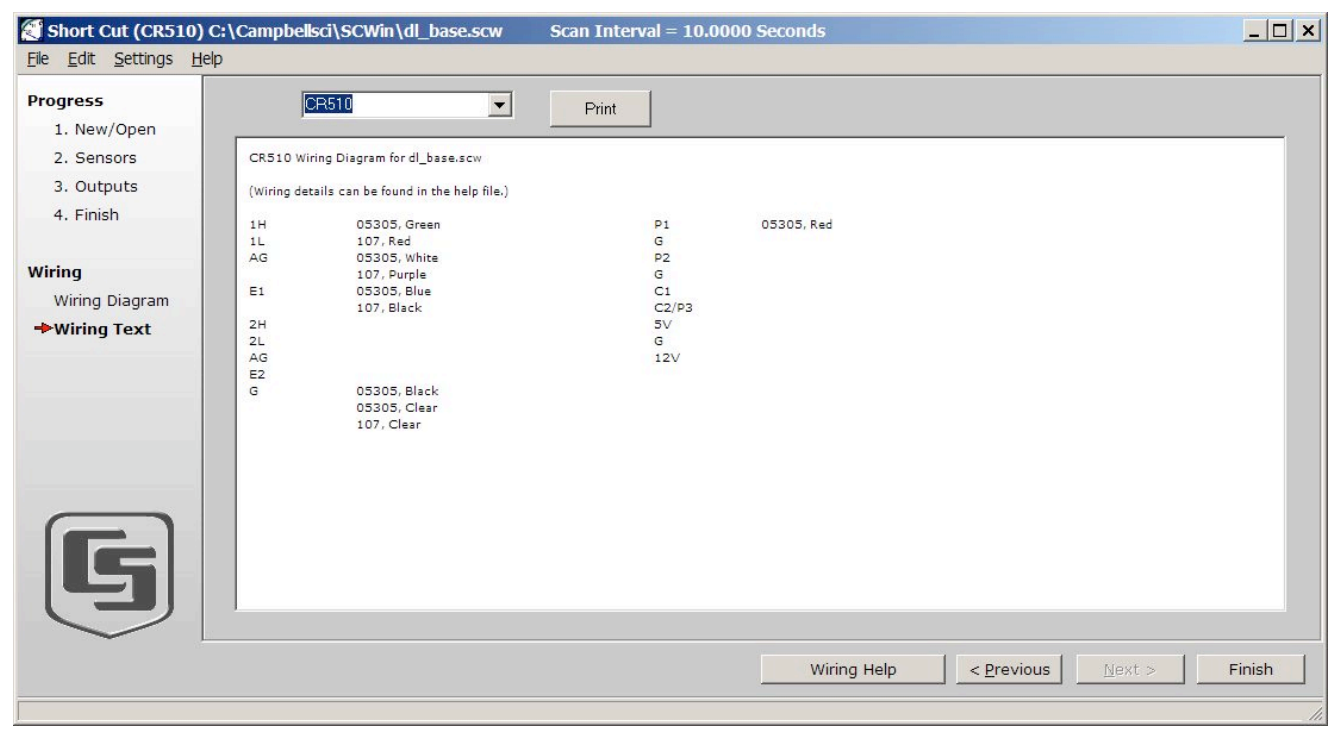

Figure 2.9. Short Cut Utility: Wiring Text Window Which Provides a List of the Data Logger Channels and the Sensor Wires Attached to Each Channel

Table 2.5. 1-min Average Data Structure (“Table 101").

\begin{tabular}{||l|l|l||}
\hline Field number & \multicolumn{1}{|c|}{ Parameter Name } & Units/field width \\
\hline \hline 1 & Line ID $(=101)$ & 3 digit \\
\hline 2 & Year & 4 digit \\
\hline 3 & Day of year & 1 to 3 digit \\
\hline 4 & Time (hhmm) & 1 to 4 digit \\
\hline 5 & Data logger ID & 1 digit \\
\hline 6 & Scalar averaged wind speed & $\mathrm{m} / \mathrm{s}$ \\
\hline 7 & Vector averaged wind speed & $\mathrm{m} / \mathrm{s}$ \\
\hline 8 & Wind direction & deg. \\
\hline 9 & Average air temperature & $\mathrm{C}$ \\
\hline 10 & Average battery voltage & $\mathrm{V}$ \\
\hline
\end{tabular}

Table 2.6. 5-min Average Data Structure (“Table 102”).

\begin{tabular}{||l|l|l||}
\hline \hline Field number & \multicolumn{1}{|c|}{ Parameter name } & \multicolumn{1}{c|}{ Units/Field Width } \\
\hline \hline 1 & Line ID (=102) & 3 digit \\
\hline 2 & Year & 4 digit \\
\hline 3 & Day of year & 1 to 3 digit \\
\hline 4 & Time (hhmm) & 1 to 4 digit \\
\hline 5 & Average battery voltage & $\mathrm{V}$ \\
\hline 6 & Minimum battery voltage & $\mathrm{V}$ \\
\hline 7 & Program signature & \\
\hline 8 & Data logger ID & 1 digit \\
\hline 9 & Scalar averaged wind speed & $\mathrm{m} / \mathrm{s}$ \\
\hline 10 & Stdev of scalar averaged wind speed & $\mathrm{m} / \mathrm{s}$ \\
\hline 11 & Vector averaged wind speed & $\mathrm{m} / \mathrm{s}$ \\
\hline 12 & Wind direction & $\mathrm{deg}$. \\
\hline 13 & Stdev wind direction & deg. \\
\hline 14 & Average air temperature & $\mathrm{C}$ \\
\hline 15 & Stdev air temperature & $\mathrm{C}$ \\
\hline
\end{tabular}




\subsection{LoggerNet Backup and Transfer to Another Computer}

It is possible to save the current incarnation of LoggerNet for backup purposes or for re-creation on another computer. During installation, LoggerNet creates a directory, C: Campbellsci (default name and location), which contains current settings for all its components along with all user-created programs and data. Only this directory needs to be saved to back up RDCDS-specific software. We recommend the following procedure to restore or transfer the LoggerNet programs and settings:

1. Save current contents of the C:lCampbellsci directory in a safe place.

2. On a computer where the LoggerNet needs to be installed or restored, make standard install of the LoggerNet from original media (CD in our case) and perform all updates and upgrades, as recommended by Campbell Scientific. For example, at the time this document was written, we had the original CD with Version 3.1 and patches to upgrade to Version 3.2.2 and Version 3.4 (obtained at the Campbell Scientific web page). The patches need to be applied sequentially after the standard install from the CD.

3. Stop all running LoggerNet applications, if any. Replace directory of C: $\backslash$ Campbellsci with a backup copy. Restart the LoggerNet server (also known as the task bar) and all necessary applications.

Please note that currently RDCDS has purchased only one license for the LoggerNet. Therefore, only one LoggerNet copy is allowed to run at a time; all other copies may be kept for backup purposes only. 


\subsection{Radio Network, Transceiver Setup and Replacement}

\subsection{General Description}

The RDCDS suit of meteorological instruments consists of the central control system, meteorological network of four remote stations, and a miniSODAR; detailed descriptions of the meteorological assets are discussed in Section 1.1 of this document. All remote instrumentation, including the miniSODAR, is connected to the central control system computer via radio links. The central control system computer serves as a controlling and data acquisition system to the entire RDCDS meteorological component. It uses two radio transceivers: one to control the network of weather stations and another for a dedicated radio link to the miniSODAR. Because most modern computers lack serial ports, we use USB-to-serial 4 port adapter to provide the two necessary serial ports on the computer.

900-MHz spread spectrum wireless data transceivers (models FGRO9CSU and FGR-115RC manufactured by FreeWave Technologies, Inc.) are used throughout the radio network. The main difference between these two models is that FGRO9CSU is a bare board radio while FGR-115RC is encased in an aluminum enclosure. We have supplied two plastic enclosures for board radios that are to be used as the miniSODAR master radio and a spare master; in the current application, all enclosed radios are interchangeable.

The radio network is designed to be capable of a "tree topology," where the center station is at the "root" of the tree and uses one "master" transceiver to connect to all weather stations, directly or through repeaters. The radio network follows a simple star layout (all "beams" are direct Point-to-Point connections) or a more complex tree design with repeaters, where connection to a station can be set up through other stations or a designated repeater; one repeater can be used to connect to several stations. Each weather station may be used as a repeater, as a measuring station, or in both capacities.

The radio link to the miniSODAR is established using two transceivers in "Point-to-Point" mode, one at the central control system (master) and the other at the miniSODAR end (slave). Because this link is a dedicated permanently active connection, neither of the transceivers can be used at any time in another link as a repeater, a master, or a slave. This allocation of dedicated link is essential to accommodate continuous data flow from the miniSODAR and to separate the weather station network from the miniSODAR.

\subsection{Radio Network Philosophy}

The meteorological radio network consists of one master and several slave transceivers at the remote sites; it may also contain designated repeaters and/or use slaves as shared repeaters. Any connection between the central and remote stations is a Point-to-Point one ${ }^{1}$. The central control system is the active side of all communication links; it initiates all connections (polls remote stations) according to the schedule programmed into the LoggerNet and collects recent data from the stations.

The meteorological master radio is set into mode 6 (Point-to-Point Slave/Master Switchable) and slaves are in mode 4 (Point-to-Point Slave/Repeater). When the master transceiver is in mode 6, it obeys 
so-called "AT-commands" from the central control system computer. These commands may contain a request to establish a connection, directly or through repeater(s), to the specific slave ID explicitly stated in the command line or referenced in the master Call Book. Our ability to schedule polling of the remote stations is based on this transceiver capability.

Each slave on the network is set into mode 4 (Point-to-Point Slave/Repeater), which allows any slave to be used as a repeater. The Call Books of slaves contain IDs of all our radios; hence, a slave will recognize any of our radios as a master and answers or relays a call, as requested. The Slave Security feature, by factory default, is turned on, so the slaves respond only to calls from a master listed in its Call Book. This combination means that if the master transceiver was replaced by another radio from our stock, any slave would still answer calls from a new master and ignore calls from all out-of-network radios.

Configuring each particular radio may seem excessive (see Section 3.3); however, this design simplifies replacing the failed transceiver with a spare or with a transceiver from another station (including the miniSODAR). It also allows a change of routing arrangements by pure software means, just by changing the corresponding calling command on the central control system computer. There is no need to visit stations and/or repeaters and reprogram radios to change the routing.

The miniSODAR link is a simple "Point-to-Point" link; it is fully transparent for a serial data stream, and no configuration is required. If a direct radio link is not feasible because of an unfavorable location of the miniSODAR and/or the central control system (buildings, distance, security, etc.), up to four repeaters may be used to circumvent obstacles. The repeaters need to be configured as usual "slaves" (see below); their serial ID numbers need to be present in the "miniSODAR" master Call Book. For the sake of uniformity, it is better to enter this routing information in lines 8 and/or 9 of the Call Book (see the section entitled, "Programming Point-to-Point Extended Call Book to Use Three or Four Repeaters" in the FreeWave Spread Spectrum Wireless Data Transceiver User Manual (FreeWave 2004). Please note that these repeaters cannot be used in any other link because the miniSODAR link is permanently "binding."

\subsection{FreeWave Radio Operation Basics}

In its current design, the central control system initiates all connections (polls remote stations). The master radio receives a Call Command from the computer using serial RS-232 interface and connects to the requested slave (via a repeater, if necessary). Mode 6 (Master/Slave Switchable For Point-To-Point Operation) allows for a variety of calling commands to be used. Evidently, any Call Command should identify addressee transceiver (slave) and a repeater path to follow (routing). A Call Book is a table that is internally stored in every FreeWave transceiver. It contains information necessary to define addressee and associated routing (for a master), or allowed connections (for a slave). The master Call Book contains a list of potential slaves with corresponding routing and an Entry to Call, a parameter that defines which slave/route to use if none was explicitly requested (default routing). The following two commands result in the master transceiver trying to establish a communication link with another transceiver:

1. ATD\#\#\#\#\#\# allows the specific radio with ID \#\#\#\#\#\#\# to be called. However, there is one caveat: the master transceiver is using the "default" routing (Repeater path) that is referenced by Entry to Call. This command is not a "direct call to specific slave" as may be understood from the FreeWave

1 The FreeWave radio also has Point-to-MultiPoint mode capability; it is not used here 
manual, but rather it is "call this specific slave using the repeater(s) referenced by Entry to Call in the Call Book and ignore slave ID in this entry."

2. ATDT\# is a request to change the Entry to Call parameter to \#, and call the slave referenced in line \# of the Call Book using the routing defined in the same line. This command requires the Repeater path to be pre-defined in the Call Book, making this a permanent routing assignment for this slave. Using permanently defined repeater paths limit our ability to choose routing without considerable reprogramming of the transceivers.

ATXC\# is a special command to change Entry to Call without actual calling out to a slave. It is similar to manually changing the Entry to Call parameter in the Call Book of the transceiver. It may be combined with the ATD\#\#\#\#\#\# command to achieve connection to the slave with ID of \#\#\#\#\#\#\# via a repeater referenced in the line \#. For a direct connection, the parameter Entry to Call must be pointed to the line that does not have a repeater path defined. Because the simple ATD\#\#\#\#\#\#\# command relies on the Entry to Call parameter, it is prudent to always precede this command with the ATXC\# command to ensure correct routing.

Our convention is to program the master Call Book so it contains all of our transceiver IDs in the Repeater table, fake IDs in the corresponding Slave section of the table, and one or two blank lines at the end of the table. Thus, combinations of ATXC\# and ATD\#\#\#\#\#\# commands allow us to call any slave (explicit indication of the slave ID in the ATD part) using any listed slave as a repeater (indirect description of the routing in the ATXC part of command) or none at all. Normally, this combination of two commands is used in the current application.

\subsubsection{Radio Programming}

A FreeWave transceiver is programmed via serial interface with the help of a standard serial cable (enclosed radios), a special interface cable (P/N ASC3610D for bare board radios), or a diagnostics cable (P/N ASC04090C). The usual sequence of steps to set up a transceiver is as follows (see the sections entitled, "Quick Start on Point-to-Point Network" and "Setting up the Transceiver Using Hyperterminal" in the FreeWave Spread Spectrum Wireless Data Transceiver User Manual (FreeWave 2004):.

1. Connect the transceiver to the serial port of a computer through appropriate serial or diagnostics cable. Make sure that the radio is powered up.

2. Start a telecommunication program that allows direct access to computer serial port, like Hyper Terminal, Minicom, or Procomm; use the following settings for the serial port: data rate to 19200, data bits to 8 , parity to none, stop bits to 1 , flow control to none.

3. Press the Setup button on the radio (on the "connector" side of radio enclosure, or on the interface cable pigtail of board radio) or, if using the diagnostics cable, press Shift-U (capital U on the computer keyboard). The three lights on the radio will turn green and the main menu will appear on the computer screen. Note that sometimes it is necessary to press 0 to invoke the main menu.

4. Make all the necessary changes to the radio configuration.

5. Exit the Setup mode by pressing the Esc key until the transceiver returns into working mode (lights turn off and/or flashing red), or power cycle the transceiver. 


\subsubsection{Master Transceiver Setup}

Two "master" radios are connected to the central control system computer. One is the master for the weather stations and the other is the master for the miniSODAR link. The weather station master is configured in mode 6 as "Master/Slave Switchable For Point-To-Point Operation" with exchange rate of 9600 baud (default exchange rate of Campbell Scientific data logger model CR510). The miniSODAR master is configured in mode 0 as "Master For Point-To-Point Operation" with exchange rate of 19200 baud (preferable exchange rate between the miniSODAR and SodarPro program). It also needs to have an additional entry in its Call Book (i.e., serial ID of the corresponding slave radio) with Entry to Call pointing on that entry. In any other respect, these master radios have the same configuration. Measures were taken to minimize reprogramming in the case of replacement of either master or any slave. A spare radio has been configured as a master for the meteorological network. It is a full "drop down" replacement for the current meteorological master and can be used as a miniSODAR master after simple re-programming (change mode from 6 to 0, exchange rate to 19200, and put the corresponding slave ID into line 8 of the Call Book, and change Entry to Call to 8). Note that current documentation only discusses changes that must be made to the default factory configuration of a radio before deployment.

Steps to program a FreeWave transceiver as meteorological master are as follows (see Section 3.3.1 and the section entitled, "Setting up a Transceiver" in the FreeWave Spread Spectrum Wireless Data Transceiver User Manual (FreeWave 2004):

1. set the Operation Mode to 6 (Point-to-Point Slave/Master Switchable)

2. set the Baud Rate to 9600

3. edit the Call Book as follows:

- enter the "bogus" serial ID number of "555-5555" for slave/master number and serial numbers of eight available transceivers as first repeaters in Entries 0 through 7 . Note that this order is very important.

- change Entry to Call to 9; now the Call Book should appear as follows:

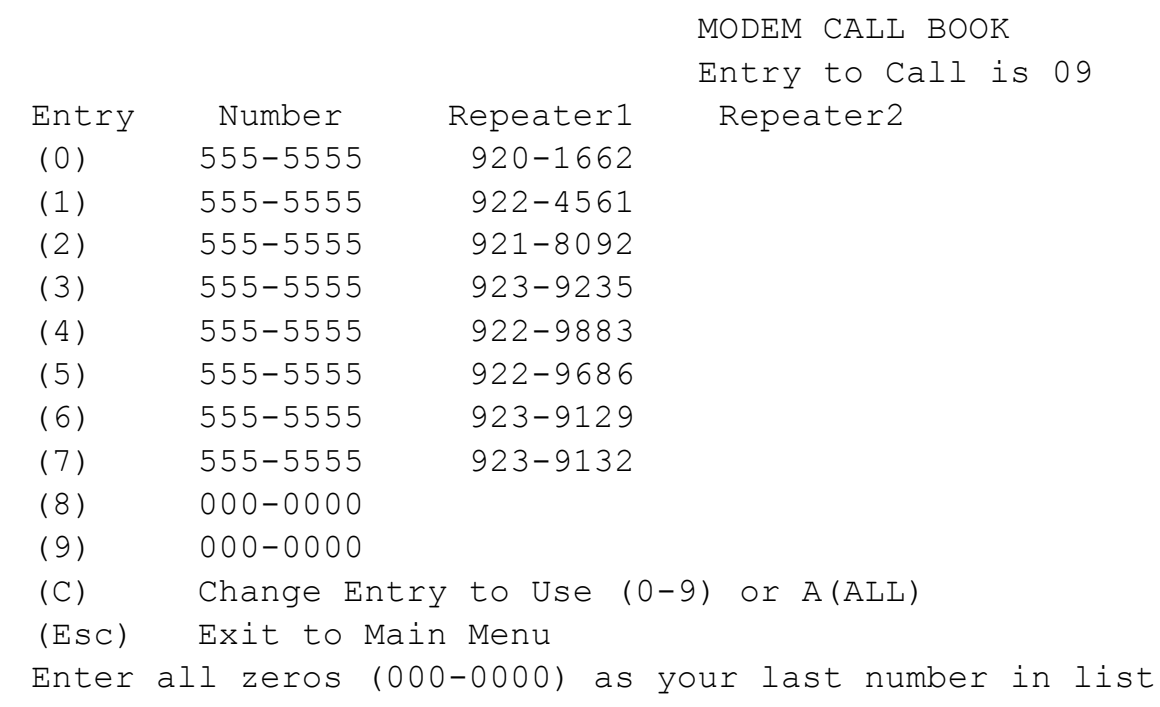


To program a FreeWave radio as miniSODAR master do the following (see Section 3.3.1 and the section entitled, "Setting up a Transceiver" in the FreeWave Spread Spectrum Wireless Transceiver User Manual (FreeWave 2004):

1. set Operation Mode to 0 (Point-to-Point Master)

2. set Baud Rate to 19200

3. edit Call Book as follows:

- enter "bogus" serial ID number of "555-5555" for slave/master number and serial numbers of 8 available transceivers as first repeaters in Entries 0 through 7 (this is not necessary for miniSODAR link, it is made for the sake of compatibility with meteorological network). Note that this order is very important

- enter the serial ID number of a corresponding "slave" radio in Entry 8 (in the current example it is 923-9132), and put no repeater (if no repeater is used) in this line

- change the Entry to Call to 8; now the Call Book should appear as follows (923-9132 is the slave transceiver at the miniSODAR end in the following example):

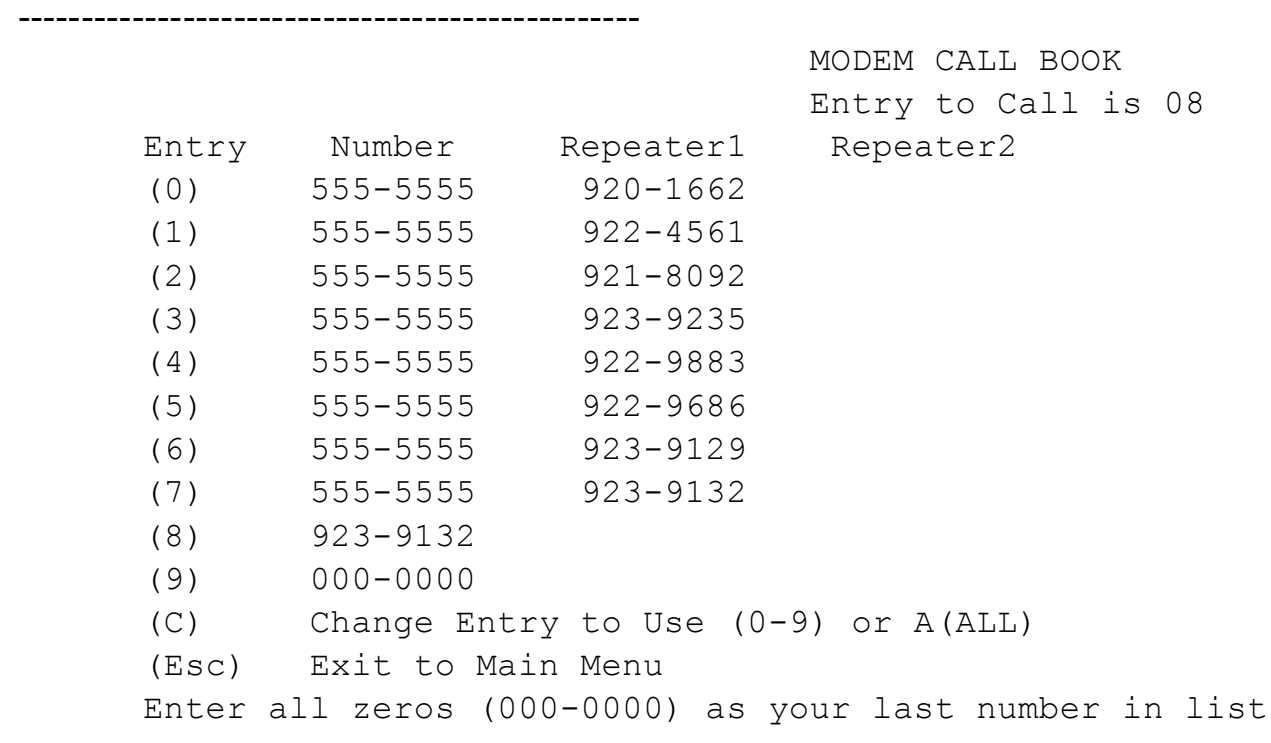

\subsubsection{Slave Transceiver Setup}

There are two kinds of "slave" radios: "miniSODAR" and "meteorological." The only difference is the Baud Rate: 9600 for meteorological and 19200 for the miniSODAR slave. All other parameters are the same. Note that the current document discusses only changes that must be made to the radio factory default configuration (see Section 3.3.1 and section entitled, "Setting up a Transceiver" in the FreeWave Spread Spectrum Wireless Transceiver User Manual (FreeWave 2004). Steps to program a FreeWave transceiver as a slave are as follows:

1. set the Operation Mode to 4 (Point-to-Point Slave/Repeater)

2. set the Baud Rate to 9600 ("meteorological") or to 19200 ("miniSODAR") 
3. edit the Call Book so it contains serial numbers of eight available transceivers, order seems to be unimportant, but it has been kept the same as in master's Call Book; now Call Book should look like that:

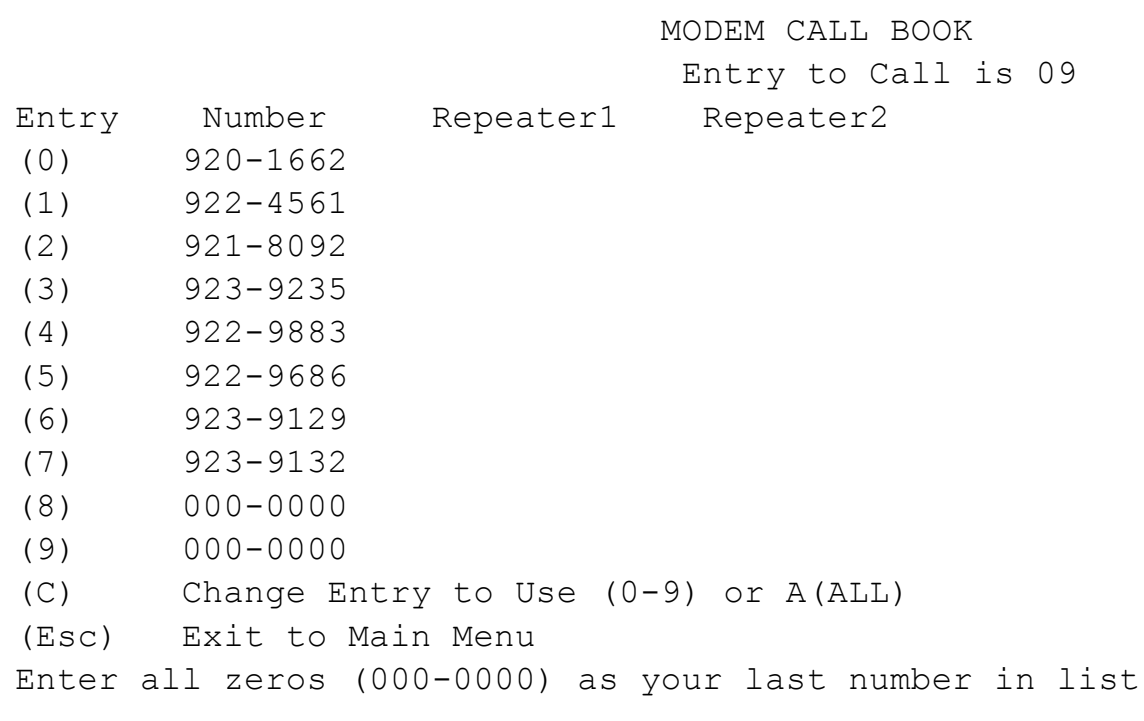

\subsection{Use with LoggerNet and SodarPro}

The central control system computer runs two programs to control remote stations and to collect data: LoggerNet for the weather station network and SodarPro for the miniSODAR. These tasks are entirely independent and, if necessary, they can be run on two separate computers with appropriate radio links.

Current radio network architecture and transceiver setup allows a certain amount of flexibility without reprogramming transceivers at remote sites. Actual radio network topology, station poll timings, routing setup, etc., are defined during the initial setup and stored in the LoggerNet software (see Section 1.0). LoggerNet usually establishes a connection to a slave radio (remote station) using the calling command line ATXCXATD\#\#\#\#\#\#, where $\mathrm{x}$ is the line in the master Call Book with a fake ID in the Number field, the requested repeater ID in the Repeater1 field (indirect addressing to the repeater), and \#\#\#\#\#\#\# is the ID of the corresponding slave (direct addressing to the slave).

Other possible calling modes include calling via a chain of repeaters. However, in this case, the Master Call Book needs to be altered as well as the LoggerNet calling command; these complicated schemes are not discussed in this document.

All calling commands are entered or modified via the "Setup" tool of the LoggerNet suite. Specific commands for a remote station are stored in the Modem property page of the Generic_ $<\mathrm{x}>$ device, where $<\mathrm{x}>$ is the remote station ID. For example, a possible command for station \#3 stored in "Generic_3 $\rightarrow$ Modem" tab can be T"ATXC2ATD9239235"R"OK"1200. This command translates as follows: connect to radio 9239235 via repeater described in Call Book line 2 and expect an "OK" response within $1200 \mathrm{~ms}$. 
Currently, the SodarPro program does not use any special feature of the transceivers. This radio link is continuously connected and is transparent to the program just like a common "wired" serial connection.

\subsection{Radio Replacement}

In the unlikely event of a transceiver failure, the radio would be replaced with another appropriately configured radio, and appropriate changes would be made in the LoggerNet and/or Call Books of other radios. If the replacement radio is taken from "our stock" (i.e., a spare, or one "borrowed" from other station), then all the programming corrections will be made at the central control system (within LoggerNet package and/or master transceiver Call Book), and there will be no need to visit other remote stations.

\subsubsection{Weather Station Network}

1. Master. Simple replacement of the failed radio with a new and properly configured radio (e.g., with a spare master radio). Note that the replacement radio should be one from our stock; otherwise, the Call Books of all slaves need to be corrected. No changes to the LoggerNet are necessary.

2. Slave. Not used as a repeater by another link. Only the calling command in the LoggerNet Setup needs to be appropriately amended: new ID into ATD\#\#\#\#\#\#\# part of the command (see Section 2.2.2).

3. Slave. Used as a repeater:

a. If the replacement radio is from our stock, only the calling commands in the LoggerNet Setup need to be appropriately amended (see Section 2.2.2). These are the command to connect to this remote station (new ID into ATD\#\#\#\#\#\#\# part), and all other commands that use this remote station as a repeater (appropriate line number into ATCX\# part).

b. If the replacement radio is not from our stock, the master Call Book must be corrected. The failed radio ID needs to be replaced with a new radio ID in the Repeater1 column. In this case, the LoggerNet Setup correction is limited only to the station calling command (new ID into ATD\#\#\#\#\#\# part).

4. Designated repeater:

a. If the replacement radio is from our stock, only the LoggerNet Setup calling commands need to be corrected (repeater defining parts, ATCX\#).

b. If the replacement radio is not from our stock, the master Call Book needs to be corrected. The failed radio ID must be replaced with a new one in the Repeater1 field of the appropriate line. No corrections to LoggerNet are necessary.

\subsubsection{MiniSODAR Link}

1. Master. Simple replacement of the failed radio with a new and properly configured radio. Note that the replacement radio should be one from our stock; otherwise, the Call Book of the miniSODAR slave radio needs to be corrected. In addition, all other slaves should be re-programmed at a later date, for consistency. 
2. Slave. If the replacement radio is from our stock, then the miniSODAR master Call Book will need to be corrected. The serial ID number of the replacement radio should be input on line 8 of the master Call Book.

3. Designated repeater. The master and slave Call Books need to be amended appropriately.

If any replacement is made, it is recommended that all transceiver Call Books remain current (all possible masters in the slave's Call Book, or all possible repeaters in the master's Call Book). New transceiver IDs will need to be put in the place of failed ones in all Call Books as soon as possible. This will maintain the designed flexibility of the network and ensure ease of maintenance in the future.

\subsection{Current Transceiver Affiliation and Configuration}

Table 3.1. List of Available Transceivers.

\begin{tabular}{||l|l|l|l|l|l||}
\hline \hline Serial ID & \multicolumn{1}{|c|}{ Mode } & \multicolumn{1}{|c|}{ Used as } & $\begin{array}{c}\text { Master Call } \\
\text { Book } \\
\text { (repeater list) }\end{array}$ & $\begin{array}{c}\text { Slave Call } \\
\text { Book (master } \\
\text { list) }\end{array}$ & \multicolumn{1}{||}{ Remarks } \\
\hline \hline $920-1662$ & $\begin{array}{l}6, \mathrm{M} / \mathrm{S} \\
\text { switchable }\end{array}$ & $\begin{array}{l}\text { Central control } \\
\text { system (met. } \\
\text { master) }\end{array}$ & 0 & 0 & $\begin{array}{l}\text { FGR-115RC, aluminum } \\
\text { enclosure }\end{array}$ \\
\hline $922-4561$ & $4, \mathrm{~S} / \mathrm{R}$ & Station \#1 & 1 & 1 & FRG-09CSU, bare board \\
\hline $921-8092$ & $4, \mathrm{~S} / \mathrm{R}$ & Station \#2 & 2 & 2 & FRG-09CSU, bare board \\
\hline $923-9235$ & $4, \mathrm{~S} / \mathrm{R}$ & Station \#3 & 3 & 3 & FRG-09CSU, bare board \\
\hline $922-9883$ & $0, \mathrm{M}$ & $\begin{array}{l}\text { Master for } \\
\text { miniSODAR }\end{array}$ & 4 & 4 & $\begin{array}{l}\text { FRG-09CSU, plastic } \\
\text { homebrewed enclosure; } 19200 \\
\text { Baud; Entry to Call = 8 }\end{array}$ \\
\hline $922-9686$ & $4, \mathrm{~S} / \mathrm{R}$ & $\begin{array}{l}\text { Spare (slave) or } \\
\text { Station \#4 }\end{array}$ & 5 & 5 & $\begin{array}{l}\text { FRG-09CSU, bare board; } \\
\text { currently set up in station \#4 }\end{array}$ \\
\hline $923-9129$ & $\begin{array}{l}6, \mathrm{M} / \mathrm{S} \\
\text { switchable }\end{array}$ & Spare (met. master) & 6 & 6 & $\begin{array}{l}\text { FRG-09CSU, plastic } \\
\text { homebrewed enclosure }\end{array}$ \\
\hline $923-9132$ & $4, \mathrm{~S} / \mathrm{R}$ & MiniSODAR slave & 7 & 7 & $\begin{array}{l}\text { FRG-09CSU, bare board; } 19200 \\
\text { Baud }\end{array}$ \\
\hline \hline
\end{tabular}

For testing purposes, on one occasion, the network was running in a crisscross fashion: station \#1 was addressed via a repeater (station \#2), station \#2 was connected via a repeater (station \#3), and station \#3 was connected via a repeater (station \#2). The corresponding calling commands were as follows:

Station \#1: T"ATXC2ATD9224561"R"OK"1200

Station \#2: T"ATXC3ATD9218092"R"OK”1200

Station \#3: T"ATXC2ATD9239235"R"OK"1200

Under normal circumstances, it is anticipated that the network would have a simple star arrangement; each remote station has a direct link with the central station. 


\subsection{The ASC miniSODAR and SodarPro Program}

\subsection{The miniSODAR Computer}

See the miniSODAR manual (Atmospheric Systems 2004) for information on general setup and operation.

The Atmospheric Systems Corporation (formerly AeroVironment) miniSODAR is based on a general-purpose VME-bus computer system (i.e., Motorola 68040 processor) that runs under the OS-9 operating system. OS-9 is a real-time, multi-task, multi-user operational system developed by Microware Systems Corporation, currently owned by RadiSys Corporation. A full set of documentation is available from the RTSI OS-9 Archive at http://os9archive.rtsi.com.

The miniSODAR computer has four serial ports. Serial port 1 is usually configured as a console (/t0 in OS-9 terms, set to $9600,8,1, \mathrm{~N}$, allows login), and serial port $2(/ \mathrm{t} 1$, set to $19200,8,1, \mathrm{~N})$ is used as a communication channel between the miniSODAR program on the VME system and the remote graphical client (i.e., SodarPro), which is run on the RDCDS central control system computer. Serial port 3 is configured for modem connection (allows incoming calls; not tested or verified).

The miniSODAR computer has complete network capabilities (TCP/IP), it allows terminal login (simple telnet style), and the system runs an ftp server when the TCP/IP network is available. It is advisable to use a network connection for SodarPro communication.

The SodarPro program on the central control system computer needs to be configured to use a serial link. This configuration is done via the menu "Station" $\rightarrow$ "PC to ASP Data I/O Settings": choose appropriate serial port (usually 5, since USB-to-serial adapter ports are mapped from COM4 to COM7, and COM4 is used by LoggerNet), 19200 Baud None Parity 8 bit Data 1 Stop bit, and check the "Connect using a serial link" box.

Because the radio link for the miniSODAR is a designated Point-to-Point link, Yagi antennas are used on both ends. To ensure the antennas are properly located during initial installation, Point-to-Point diagnostics software from FreeWave Technologies, Inc, can be used.

\subsection{SodarPro Peculiarities}

SodarPro is a Windows-based remote client that allows remote control over the miniSODAR (configuration, time synchronization, data collection/retrieval, but not a terminal login) and provides certain graphical displays of the data collected. It can use serial link or network (TCP/IP) connection to the miniSODAR computer. Unfortunately, the SodarPro documentation is not current and is several versions old. Some results of empirical observations of SodarPro performance are as follows:

1. SodarPro sometimes crashes without the slightest provocation or warning, and it may leave the following error message: SodarPro - [ (6.21) rdcd_1-DFS] : SodarPro.exe Application Error: The instruction at "0x73dd439a" referenced memory at "0x00000008." The memory could not be "read." Restart of the SodarPro usually corrects the problem 
2. after restart, it sometimes forgets which serial port to use and yet gives no error message in this case, just sits indefinitely doing nothing. A properly configured serial link (see Section 4.1) solves this problem

3. after restart, it may forget its display options (our usual choice for the upper-left quadrant is recent wind table, the lower left is wind profiles, the upper right is wind barb plot, and the lower right is a quasi-facsimile, so-called DFS chart) that needs to be rearranged manually

4. it may display an incorrect miniSODAR or station name on its window. 


\subsection{Central Control System and Weather Stations}

\subsection{The Central Control System, Tools, and Spare Parts}

The central control system is not a "station" per se, but rather a laptop computer connected to two radio transceivers. All components of the central control system are listed in Table 5.1.

Table 5.1. Central Control System Part List

\begin{tabular}{|c|c|c|c|}
\hline Part & Model & Vendor & Remarks \\
\hline Laptop & Dell Latitude & Dell & $\begin{array}{l}\text { WinXP laptop with LoggerNet and } \\
\text { MiniSODARPro software installed } \\
\text { and properly configured }\end{array}$ \\
\hline USB-to-serial 4-port adapter & $\begin{array}{l}\text { Keyspan USA- } \\
\text { 94WLC }\end{array}$ & Keyspan & $\begin{array}{l}\text { Needed to provide necessary at } \\
\text { least } 2 \text { serial ports }\end{array}$ \\
\hline $\begin{array}{l}900-\mathrm{MHz} \text { spread spectrum } \\
\text { wireless data transceiver with } \\
\text { power supply }\end{array}$ & FGR-115RC & $\begin{array}{l}\text { FreeWave } \\
\text { Technologies, Inc. }\end{array}$ & $\begin{array}{l}\text { Meteorological network master } \\
\text { radio; wall type } 120 \text { VAC to } 12 \\
\text { VDC adapter }\end{array}$ \\
\hline $\begin{array}{l}900 \mathrm{MHz} \text { spread spectrum } \\
\text { wireless data transceiver with } \\
\text { power supply }\end{array}$ & FGRO9CSU & $\begin{array}{l}\text { FreeWave } \\
\text { Technologies, Inc. }\end{array}$ & $\begin{array}{l}\text { MiniSODAR serial link master } \\
\text { radio; bare board transceiver } \\
\text { installed into home brewed plastic } \\
\text { enclosure; wall type } 120 \text { VAC to } \\
12 \text { VDC adapter }\end{array}$ \\
\hline Omni-directional antenna & FG9026 & Antenex & $\begin{array}{l}\text { Antenna for meteorological } \\
\text { network }\end{array}$ \\
\hline Antenna cable & $\begin{array}{l}\text { ASC0504NN } \\
\text { WRM111505 }\end{array}$ & $\begin{array}{l}\text { FreeWave } \\
\text { Technologies, Inc. }\end{array}$ & $50^{\prime} \mathrm{N}$ male to $\mathrm{N}$ male $\mathrm{RF}$ cable \\
\hline Yagi antenna & YA5900W & $\begin{array}{l}\text { FreeWave } \\
\text { Technologies, Inc. } \\
\text { (Radial/Larsen antenna } \\
\text { technologies) }\end{array}$ & $\begin{array}{l}\text { Antenna for miniSODAR serial } \\
\text { link. Yagi } 7 \text { element, } 11 \mathrm{dBi}, 890 \text { - } \\
960 \mathrm{MHz}, \mathrm{N} \text { female connector }\end{array}$ \\
\hline Antenna cable & $\begin{array}{l}\text { ASC0504NN } \\
\text { WRM111505 }\end{array}$ & $\begin{array}{l}\text { FreeWave } \\
\text { Technologies, Inc. }\end{array}$ & $50^{\prime} \mathrm{N}$ male to $\mathrm{N}$ male $\mathrm{RF}$ cable \\
\hline $\begin{array}{l}\text { Two "standard" serial 9-pin to } \\
\text { 9-pin cables }\end{array}$ & & & $\begin{array}{l}\text { One is for the meteorological } \\
\text { network and the other is for the } \\
\text { miniSODAR serial link. }\end{array}$ \\
\hline
\end{tabular}

A tools and spare part kit should be stored with the central control system; its part list is presented in Table 5.2. 
Table 5.2. Tools and Spare Part Kit

\begin{tabular}{|c|c|c|c|}
\hline Part & Model & Vendor & Remarks \\
\hline Laptop & Dell Latitude & Dell & $\begin{array}{l}\text { Spare computer, "drop down" replacement } \\
\text { for the laptop at the central control system; } \\
\text { WinXP laptop with LoggerNet and } \\
\text { SodarPro software installed and properly } \\
\text { configured }\end{array}$ \\
\hline $\begin{array}{l}\text { USB-to-serial 4-port } \\
\text { adapter }\end{array}$ & $\begin{array}{l}\text { Keyspan USA- } \\
\text { 94WLC }\end{array}$ & Keyspan & \\
\hline $\begin{array}{l}\text { 900-MHz spread } \\
\text { spectrum wireless data } \\
\text { transceiver with power } \\
\text { supply }\end{array}$ & FGRO9CSU & $\begin{array}{l}\text { FreeWave } \\
\text { Technologies, Inc. }\end{array}$ & $\begin{array}{l}\text { Spare master radio; bare board transceiver } \\
\text { installed into home brewed plastic } \\
\text { enclosure and configured as meteorological } \\
\text { network master }\end{array}$ \\
\hline Omni-directional antenna & FG9026 & $\begin{array}{l}\text { Antenex (FreeWave } \\
\text { Technologies, Inc.) }\end{array}$ & Spare antenna for meteorological network \\
\hline MiniSODAR serial cable & & & RJ-45 to D-9 (standard serial) cable \\
\hline Toolbox & & & $\begin{array}{l}\text { Tools necessary to assemble/disassemble } \\
\text { remote stations }\end{array}$ \\
\hline GPS unit & eTrex Summit & Garmin & $\begin{array}{l}\text { For GPS-based positioning, height, and } \\
\text { orientation }\end{array}$ \\
\hline Manuals & & & $\begin{array}{l}\text { Collection of manuals for all parts and } \\
\text { sensors used }\end{array}$ \\
\hline $\begin{array}{l}\text { Variety of network and } \\
\text { serial cables, null } \\
\text { modems, gender } \\
\text { changers, etc. }\end{array}$ & & & \\
\hline
\end{tabular}

\subsection{Weather Station Sensors and Parts}

Each weather station consists of a tripod; an enclosure that houses a data logger, a radio transceiver, and a battery charger; a solar panel; wind and temperature sensors; and internal and external batteries (see Table 5.3) 
Table 5.3. Weather Station Part List

\begin{tabular}{|c|c|c|c|}
\hline Part & Model & Vendor & Remarks \\
\hline Data logger & CR510 & Campbell Scientific & $\begin{array}{l}\text { Should have standard Array Based } \\
\text { OS (not Park Bus OS) }\end{array}$ \\
\hline $\begin{array}{l}\text { Wind speed and } \\
\text { direction sensor }\end{array}$ & $\begin{array}{l}\text { RM Young } 05305-A Q \\
\text { Wind Monitor Wind } \\
\text { Speed and Direction } \\
\text { Sensor }\end{array}$ & Campbell Scientific & \\
\hline Air temperature & 107 Temperature Probe & Campbell Scientific & \\
\hline $\begin{array}{l}\text { Radiation shield for a } \\
\text { temperature probe }\end{array}$ & $41303-5 \mathrm{~A}$ & Campbell Scientific & 6 Plate Gill radiation shield \\
\hline $\begin{array}{l}\text { Power Supply and } \\
\text { Charging Regulator }\end{array}$ & PS100 & Campbell Scientific & \\
\hline Solar panel & SP10 & Campbell Scientific & \\
\hline CSI to RS232 interface & SC105 & Campbell Scientific & $\begin{array}{l}\text { For connection of a data logger to a } \\
\text { standard serial (RS-232) device }\end{array}$ \\
\hline Enclosure & $\begin{array}{l}\text { ENC12/14, \#5961, } \\
\text { option -LM }\end{array}$ & Campbell Scientific & $\begin{array}{l}\text { Medium sized enclosure with one } \\
\text { sealable conduit (\#5961); mounting } \\
\text { brackets option -LM attaches the } \\
\text { enclosure to the leg base of a } \\
\text { CM110 tripod }\end{array}$ \\
\hline $\begin{array}{l}900 \mathrm{MHz} \text { spread } \\
\text { spectrum wireless data } \\
\text { transceiver }\end{array}$ & FGRO9CSU & $\begin{array}{l}\text { FreeWave } \\
\text { Technologies, Inc. }\end{array}$ & \\
\hline Yagi antenna & YA5900W & $\begin{array}{l}\text { FreeWave } \\
\text { Technologies, Inc. } \\
\text { (Radial/Larsen antenna } \\
\text { technologies?) } \\
\end{array}$ & $\begin{array}{l}\text { Antenna for miniSODAR serial } \\
\text { link. Yagi } 7 \text { element, } 11 \mathrm{dBi}, 890 \text { - } \\
960 \mathrm{MHz}, \mathrm{N} \text { female connector }\end{array}$ \\
\hline Antenna cable & $\begin{array}{l}\text { ASC0504NN } \\
\text { WRM111505 }\end{array}$ & $\begin{array}{l}\text { FreeWave } \\
\text { Technologies, Inc. }\end{array}$ & $50^{\prime} \mathrm{N}$ male to $\mathrm{N}$ male $\mathrm{RF}$ cable \\
\hline $12 \mathrm{~V}$ internal battery & BP-12 & Campbell Scientific & $\begin{array}{l}\text { Small sealed battery; enough for } 15 \\
\text { hrs of operation }\end{array}$ \\
\hline $\begin{array}{l}12 \mathrm{~V} \text { deep cycle battery } \\
\text { (car or marine) }\end{array}$ & & & $\begin{array}{l}\text { To be purchased and disposed of at } \\
\text { every deployment to avoid } \\
\text { hazardous material shipment } \\
\text { hassles (optional) }\end{array}$ \\
\hline Battery container & $\begin{array}{l}\text { Any type, approved for } \\
\text { the above battery }\end{array}$ & & \\
\hline Tripod & CM-110 & Campbell Scientific & $\begin{array}{l}\text { 10-Foot Aluminum Instrument } \\
\text { Tripod }\end{array}$ \\
\hline
\end{tabular}

\subsection{Enclosure Layout and Wiring}

Each weather station enclosure was modified (holes drilled, inlets punched, connectors installed, wiring soldered, etc.). Connectors used are AMPHENOL, except RF antenna connector that was provided by East Coast Microwave (see Table 5.4). 
Table 5.4. Weather Station Enclosure Connectors

\begin{tabular}{||l|c|c|c|c|l||}
\hline \hline $\begin{array}{l}\text { Connection } \\
\text { designation }\end{array}$ & $\begin{array}{c}\text { Number } \\
\text { of pins }\end{array}$ & $\begin{array}{c}\text { Gender } \\
\text { (cable end) }\end{array}$ & $\begin{array}{c}\text { Part number } \\
\text { (cable) }\end{array}$ & $\begin{array}{c}\text { Part number } \\
\text { (enclosure) }\end{array}$ & \multicolumn{1}{||}{ Remarks } \\
\hline \hline Anemometer & 6 & Male & $97-3106 \mathrm{~A}-14 \mathrm{~S}-6 \mathrm{P}$ & $97-3102 \mathrm{~A}-14 \mathrm{~S}-6 \mathrm{~S}$ & \\
\hline $\begin{array}{l}\text { Temperature } \\
\text { sensor }\end{array}$ & 4 & Male & $97-3106 \mathrm{~A}-14 \mathrm{~S}-2 \mathrm{P}$ & $97-3102 \mathrm{~A}-14 \mathrm{~S}-2 \mathrm{~S}$ & $\begin{array}{l}\text { Pigtail inside the enclosure } \\
\text { has a resistor divider hidden } \\
\text { in it. }\end{array}$ \\
\hline Solar panel & 2 & Female & $97-3106 \mathrm{~A}-12 \mathrm{~S}-3 \mathrm{~S}$ & $97-3102 \mathrm{~A}-12 \mathrm{~S}-3 \mathrm{P}$ & \\
\hline Battery & 2 & Male & $97-3106 \mathrm{~A}-12 \mathrm{~S}-3 \mathrm{P}$ & $97-3106 \mathrm{~A}-12 \mathrm{~S}-3 \mathrm{~S}$ & \\
\hline RF antenna & $\begin{array}{l}\text { Coaxial, } \\
\text { circular }\end{array}$ & Female & $\mathrm{n} / \mathrm{a}$ & 03 R6316-56-006B & $\begin{array}{l}\text { Pigtail; one end is "big” box } \\
\text { mountable female N-type } \\
\text { connector, the other is cable } \\
\text { male SMA-type connector. }\end{array}$ \\
\hline \hline
\end{tabular}

The remote station connector layout (bottom side of the enclosure, bottom view from the outside) and inlet sizes are shown in Figure 5.1 and in the following diagram:

Solar panel

2-pin, male, 17.5

$\mathrm{mm}$ round, $\approx 3 / 4$ "

chassis puncher

External battery

2-pin, female, 17.5

$\mathrm{mm}$ round, $\approx 3 / 4$ "

chassis puncher
Temperature

4-pin, female, 19.2

$\mathrm{mm}$ round, $\approx 3 / 4$ "

chassis puncher
Wind speed and

direction

6-pin, female, $19.2 \mathrm{~mm}$

round, $\approx 3 / 4$ " chassis

puncher
Radio

Coaxial, pigtail,

$13 \times 16 \mathrm{~mm}$ oval, $\approx 1 / 2 "$

chassis puncher 


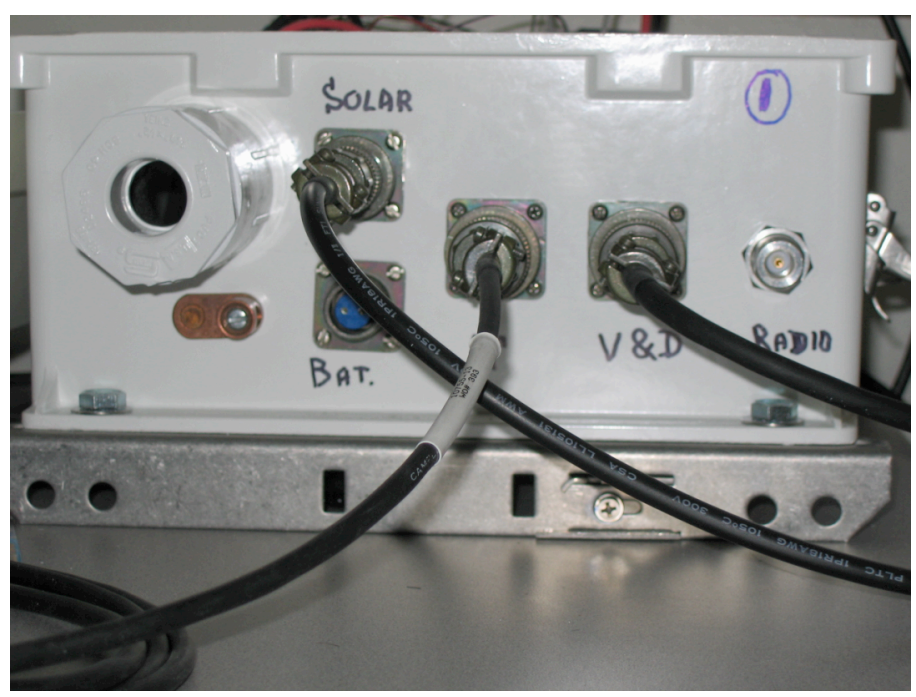

Figure 5.1. Bottom View of a Weather Station Enclosure

The internal wiring of the enclosure was made with pieces of bare end cables cut from corresponding sensors (a cable was cut approx $12 \mathrm{in}$. from the bare wire end and a pair of connectors was soldered into it. Box sockets were then installed into the enclosure). The connector pinout and wire color are listed in Table 5.5-Table 5.8.

Table 5.5. Wind Speed and Direction Sensor Connector Pinout and Wire Color Coding

\begin{tabular}{|l|l|l|l|l||}
\hline \hline Pin & \multicolumn{1}{|c|}{ Color (cable) } & \multicolumn{1}{|c|}{ Color (inside) } & \multicolumn{1}{|c||}{ Signal name } & \multicolumn{1}{c||}{ Destination } \\
\hline \hline A & White & White & AG & CR510/AG \\
\hline B & Red & Red & Pulse & CR510/P1 \\
\hline C & Green & Green & Sig+ (Analog) & CR510/SE1 \\
\hline D & Blue & Blue & Excitation & CR510/E1 \\
\hline E & Black & Black & Ground & CR510/G \\
\hline F & Clear & Clear & Shield & CR510/G \\
\hline \hline
\end{tabular}

Table 5.6. Temperature Sensor Connector Pinout and Wire Color Coding. (Note that the bare wire tail of the cable has four wires, while the cable itself has two signal wires and a shield; pigtail inside the enclosure has a resistor divider concealed in it).

\begin{tabular}{|l|l|l|l|l|l||}
\hline \hline Pin & \multicolumn{1}{|c|}{ Color (cable) } & \multicolumn{1}{|c|}{ Color (inside) } & \multicolumn{1}{|c|}{ Signal name } & \multicolumn{1}{|c|}{ Tail wire color } & \multicolumn{1}{|c|}{ Destination } \\
\hline \hline A & White & White & T & Red & CR510/SE2 \\
\hline B & Black & Black & Excitation & Black & CR510/E1 \\
\hline C & Shield & Shield & Shield & Clear & CR510/G \\
\hline D & N/C & N/C & Signal ground & Purple & CR510/AG \\
\hline \hline
\end{tabular}


Table 5.7. External Battery Connector Pinout and Wire Color Coding.

\begin{tabular}{||l|l|l|c|l||}
\hline \hline Pin & Color (cable) & Color (inside) & Signal name & Destination \\
\hline \hline A & Red & Red & + & Charger/Ext. batt. \\
\hline B & Black & Black & - & Charger/Ext. batt. \\
\hline
\end{tabular}

Table 5.8. Solar Panel Connector Pinout and Wire Color Coding.

\begin{tabular}{||l|l|l|c|l||}
\hline \hline Pin & Color (cable) & Color (inside) & Signal name & \multicolumn{1}{|c||}{ Destination } \\
\hline \hline A & Red & Red & + & Charger/CRG \\
\hline B & Black & Black & - & Charger/CRG \\
\hline \hline
\end{tabular}

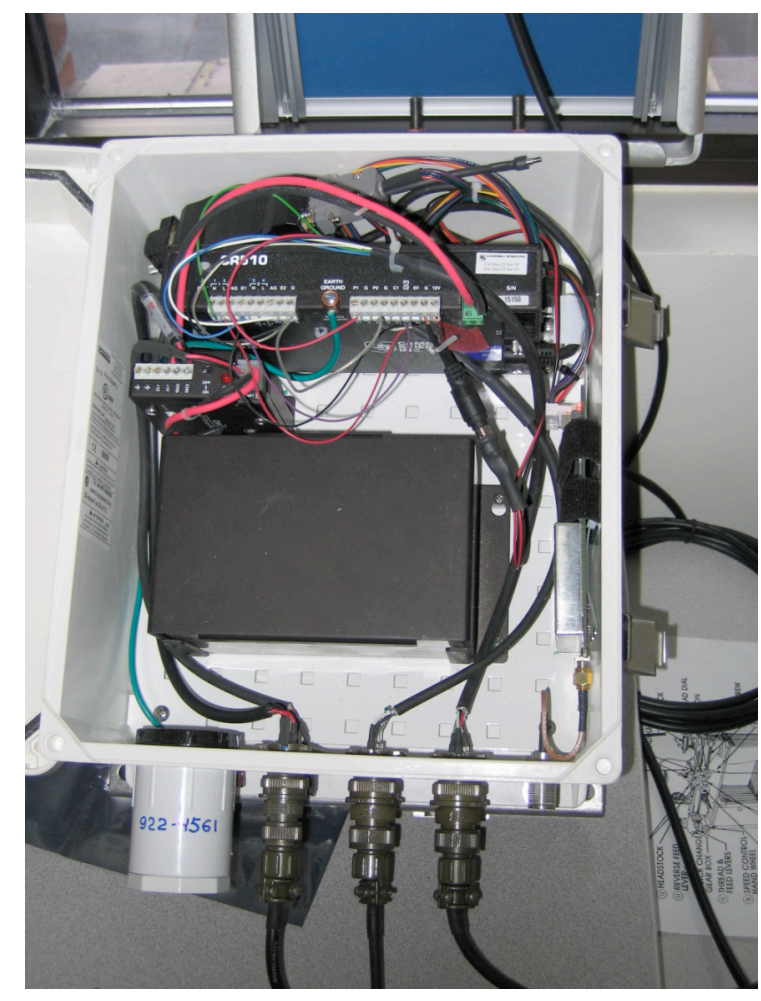

Figure 5.2. Inside View of the Enclosure. The large black rectangle is an internal battery. 


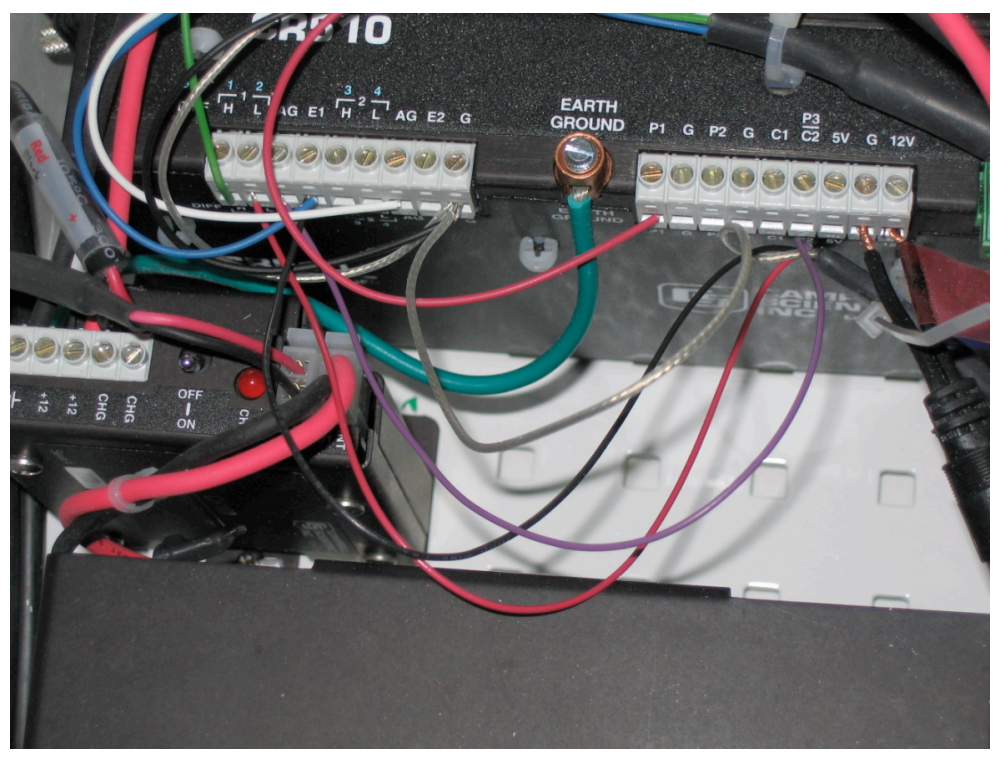

Figure 5.3. Close View of the Data Logger Contact Panel and the Power Supply (battery charger) 



\subsection{Operation of RDCDS Meteorological Component}

After installing all equipment in the field and at the central control system, the Component is ready for operation. All surface weather stations and the miniSODAR should be switched on as a part of the installation procedure (see RDCDS Meteorological Component Quick Installation Guide). The LoggerNet and SodarPro software should start automatically when the laptop computer is turned on. Several windows usually open on the laptop (see Figure 6.1): the LoggerNet Toolbar (upper right), the LoggerNet RTMC-RT (middle right), the LoggerNet Status Monitor (lower right), and the SodarPro (upper left). Note that the LoggerNet retains a list of windows and their positions from the previous session. Therefore, if the RTMC-RT or Status Monitor windows were closed at the end of the previous session, they need to be opened using the corresponding icon on the Toolbar.

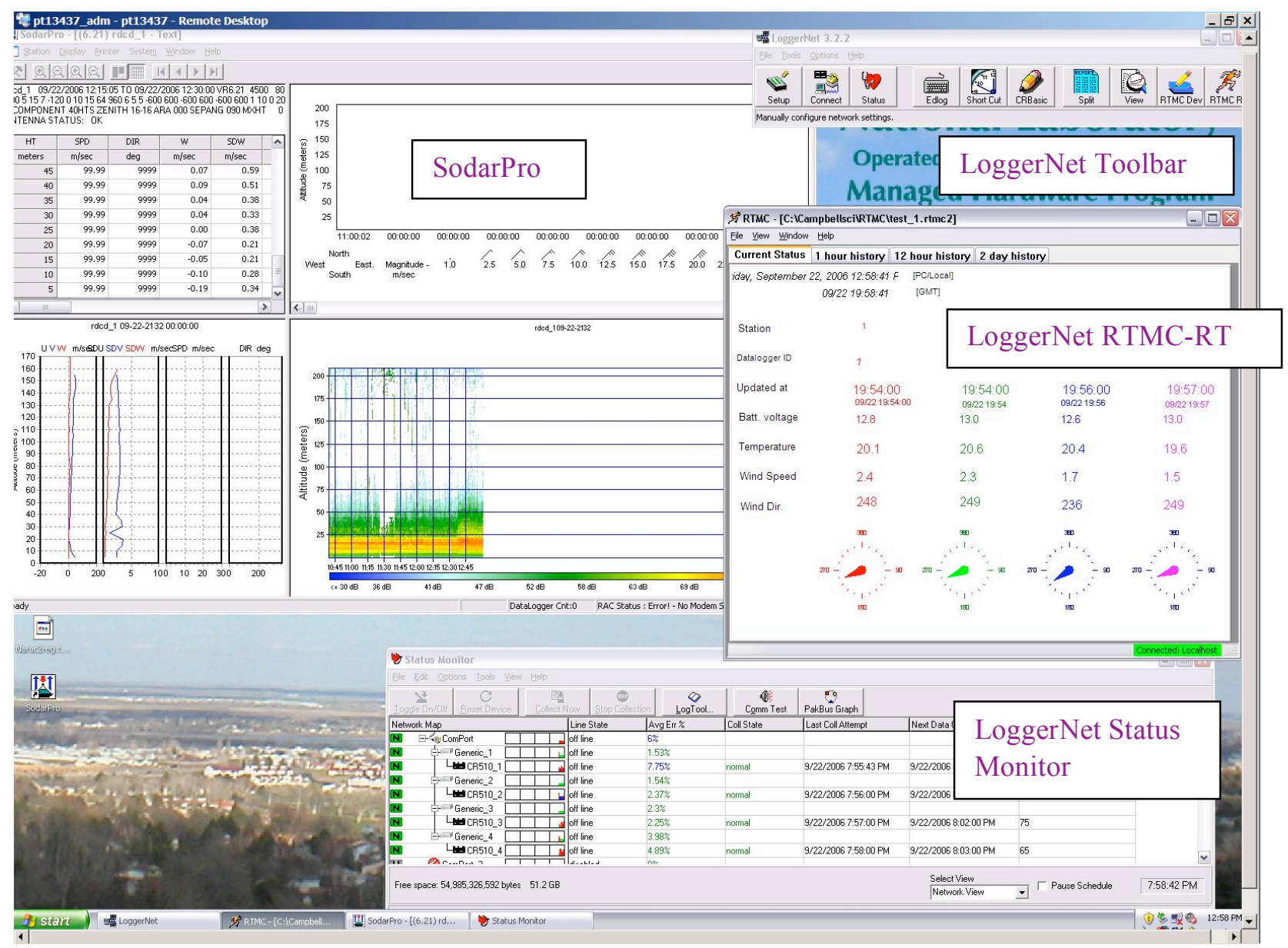

Figure 6.1. Laptop Screen with LoggerNet and SodarPro Windows. Purple labels have been added or clarity, and are not part of the display

The LoggerNet Server opens the Toolbar during its operation. If the Toolbar is closed, then the Server stops operating (no communications or data retrieval). This does not affect data collection at the remote weather stations; all backlogged data are retrieved automatically at the next LoggerNet startup and 
displayed at the RTMC-RT. Content descriptions of each window can be found in the LoggerNet documentation.

SodarPro is a software client that allows remote control over the miniSODAR, real time data transfer, and graphical display of the data collected on the laptop screen. However, the miniSODAR will operate without SodarPro running; the data are collected and stored locally at the miniSODAR computer; the data can be retrieved using SodarPro or other standard means of file transfer (e.g., FTP). Note that SodarPro does not display historical data; it shows the data collected after the most recent startup.

If for some reason LoggerNet and/or SodarPro have to be restarted, they can be stopped by closing the Toolbar and/or SodarPro windows and started again using the corresponding icons on the laptop desktop. 


\subsection{Replacement of Failed Parts}

The main goal of the spare part kit is to minimize efforts and down time that might be necessary to restore full functionality to the RDCDS.

\subsection{Weather Stations}

The Component has one spare weather station that is intended as a replacement if any failure occurs at a remote station (sensor, data logger, solar panel, etc.). All troubleshooting and repairs can be conducted later. The LoggerNet software is configured to monitor and poll all four stations. Therefore, the "end user" needs to adjust data assimilation procedures to disregard failed station and include spare one. This spare station also can be used as a fourth remote station, a radio network repeater, or both.

If the radio network is configured to use a repeater(s) and a failed station was used as a repeater, it is necessary to change calling commands of all the affected stations to address the change of repeater (Dial Script value in Generic_ID $\rightarrow$ Modem property page; see Sections 2.0 and 3.0).

If a radio transceiver has failed, and it is impractical to replace the entire station, a transceiver can be replaced with a spare. The spare transceiver should be properly configured and all calling commands (Dial Script value in Generic_ID $\rightarrow$ Modem property page) that used that transceiver as a repeater or a slave should be amended accordingly. Further details can be found in Sections 2.0 and 3.o.

In the case of a failed meteorological sensor in situations where it is unpractical to replace the entire station, the failed sensor can be replaced with another sensor from a spare station; no changes in hardware or software are necessary. If the replacement sensor is not form our stock, it needs to be either supplied with an appropriate cable connector or directly wired: the bare wire cable end is to be put through a conduit hole in the enclosure, and wires are to be connected to the data logger contact panel (see Section 5.3 for details).

\subsection{Central Control System}

The central control system spare part set contains several parts that are direct replacements ("drop down," need no configuration or initialization): a laptop, an omni-directional antenna, a USB-to-serial adapter, and a meteorological network master radio transceiver. These spares are either copies of the working parts (e.g., USB-to-serial adapter) or full functional analogs (e.g., master radio, which is a slightly different model housed in a homemade enclosure). The meteorological master radio spare can also be used as a replacement for the miniSODAR master or any slave after certain re-programming is completed (see Section 3.0). 



\subsection{Glossary}

ATD

ATXC

Call Book

Central Control System

ComPort

Connect

CR510 device

Dial script

Entry to Call

FreeWave Spread Spectrum Wireless Data Transceiver

Generic Modem

LoggerNet

MINISODAR, miniSODAR
One of modem-like AT-commands used to control FreeWave Transceiver; establishes link with specific slave (ID must follow the command) via default router(s) (listed in the Call Book line referenced by Entry to Call).

One of modem-like AT-commands used to control FreeWave Transceiver; instructs the transceiver to change Entry to Call parameter.

An internal table stored in a FreeWave Transceiver that contains IDs of master (slave) transceivers on the network, and corresponding routing information.

A combination of components that allow for data collection, data storage, and communications with the weather stations and sodar.

A pre-defined logical device in the Setup utility (part of the LoggerNet software); ComPort is used control serial communications via standard serial port (RS232).

A utility in the LoggerNet software to check out and debug connection to a remote data logger.

A pre-defined logical device in the Setup utility (part of the LoggerNet software) that is used to control Campbell Scientific Data Logger Model CR510.

A command line (stored on Hardware property page of a Generic Modem device in the Setup utility) to be sent to the weather stations master radio to setup connection with specific weather station; aggregate of Dial Scripts from all Generic Modems defines a topology of radio network.

A parameter in the Call Book pointing to the line containing default routing information.

A 900-MHz spread spectrum transceiver designed for serial communications.

A pre-defined logical device in the Setup utility (part of the LoggerNet software); it is a general-purpose communication device or modem emulator.

A suite of software tools to develop and run a network of meteorological instruments.

Sonic Detection And Ranging. A surface-based remote sounding device to measure wind profile in the lower atmosphere.
Sections 2.2 .2 and 3.3

Sections 2.2 .2 and 3.3

Section 1.0

Sections 1.1, 1.0, and 5.1

Section 2.2.1

Section 2.1

Section 0

Sections 2.2 .2 and 1.0

Section 1.0

Section 1.0

Section 2.2.2

Section 2.1

Sections 1.1 .2 and 1.0 
Modem property page

Rapidly Deployable Chemical Defense System (RDCDS)

Real Time Monitor and Control Development (RTMC Dev)

Real Time Monitor and Control Development RunTime (RTMC-RT)

Setup

Short Cut

SodarPro

Status Monitor

Toolbar
A page in a Generic Modem device properties (Setup utility) that contains "Dial Script" line that defines remote station to be called.

A system that has been developed in the DHS to help protect against indoor and outdoor releases of toxic industrial chemicals or chemical warfare agents.

A utility in the LoggerNet software used to create a graphical display of collected data.

A utility in the LoggerNet software used to show a graphical display prepared with the RTMC Device utility.

A utility in the LoggerNet software used to setup and configure the network.

A utility in the LoggerNet software used to create, upload, and compile a program on a data logger.

A Windows-based remote client to control over the miniSODAR (configuration, time synchronization, data collection/retrieval) and real-time display of the data collected.

A utility in the LoggerNet software to monitor current status of all network links.

Also known as the "LoggerNet Server"; main program in the LoggerNet software, performs communications with the devices on the network, and places a toolbar on the computer screen to initiate and navigate through other applications.
Section 2.2.2

Section 1.0

Section 2.1

Section 2.1

Section 2.1

Section 2.1

Section 1.0

Section 2.1

Section 2.1 


\subsection{References}

Atmospheric Systems, 2004: Doppler MiniSODAR System, Operation and Maintenance Manual. Atmospheric Systems Corporation.

Berg, L. K., and M. S. Pekour, 2007: RDCDS Meteorological Component Quick Installation Guide. PNNL-XXXXX.

FreeWave Technologies, 2004: Spread Spectrum Wireless Data Transceiver User Manual, Version 6.3. FreeWave Technologies, Inc.

U.S. Environmental Protection Agency, 1987: Ambient Monitoring Guidelines for Prevention of Significant Deterioration, EPA-450/4-87-007.

U.S. Nuclear Regulatory Commission, 2007: Regulatory Guide 1.23, Meteorological Monitoring Programs for Nuclear Power Plants. Office of Nuclear Regulatory Research 

